\title{
On Lipschitz Continuity of Projections onto Polyhedral Moving Sets
}

\author{
Ewa M. Bednarczuk ${ }^{1}$ (D) Krzysztof E. Rutkowski ${ }^{2,3}$
}

Published online: 11 September 2020

(c) The Author(s) 2020

\begin{abstract}
In Hilbert space setting we prove local lipchitzness of projections onto parametric polyhedral sets represented as solutions to systems of inequalities and equations with parameters appearing both in left- and right-hand sides of the constraints. In deriving main results we assume that data are locally Lipschitz functions of parameter and the relaxed constant rank constraint qualification condition is satisfied.
\end{abstract}

Keywords Lipschitzness of projection - Relaxed constant rank constraint qualification condition · Lipschitz-likeness · Graphical subdifferential mapping

Mathematics Subject Classification 47N10 - 49J52 - 49J53 · 49K40 • 90C31

\section{Introduction}

Continuity of metric projections of a given $\bar{v}$ onto moving subsets have already been investigated in a number of instances. In the framework of Hilbert spaces, the projection $P_{C}(\bar{v})$ of $\bar{v}$ onto closed convex sets $C, C^{\prime}$, i.e., solutions to optimization problems

$$
\text { minimize }\|z-\bar{v}\| \text { subject to } z \in C \text {, }
$$

$凶$ Krzysztof E. Rutkowski

k.rutkowski@uksw.edu.pl

Ewa M. Bednarczuk

Ewa.Bednarczuk@ibspan.waw.pl

1 Faculty of Mathematics and Information Science, Warsaw University of Technology, Warsaw, Poland

2 Faculty of Mathematics and Natural Sciences, School of Exact Sciences, Cardinal Stefan Wyszyński University, Warsaw, Poland

3 Systems Research Institute of the Polish Academy of Sciences, Warsaw, Poland 
are unique and Hölder continuous with the exponent $1 / 2$ in the sense that there exists a constant $\ell_{H}>0$ with

$$
\left\|P_{C}(\bar{v})-P_{C^{\prime}}(\bar{v})\right\| \leq \ell_{H}\left[d_{\rho}\left(C, C^{\prime}\right)\right]^{1 / 2}
$$

where $d_{\rho}(\cdot, \cdot)$ denotes the bounded Hausdorff distance (see [2] and also [8, Example 1.2]).

In the case where the sets, on which we project a given $\bar{v}$, are solution sets to systems of equations and inequalities, the problem Proj is a special case of a general parametric problem

$$
\begin{aligned}
& \operatorname{minimize} \varphi_{0}(p, x) \text { subject to } \\
& \varphi_{i}(p, x)=0 \quad i \in I_{1}, \quad \varphi_{i}(p, x) \leq 0 \quad i \in I_{2},
\end{aligned}
$$

where $x \in \mathcal{H}, p \in \mathcal{D} \subset \mathcal{G}, \mathcal{H}$-Hilbert space, $\mathcal{G}$-metric space, $\varphi_{i}: \mathcal{D} \times \mathcal{H} \rightarrow \mathbb{R}$, $i \in\{0\} \cup I_{1} \cup I_{2}$. There exist numerous results concerning continuity of solutions to problem (Par) in finite dimensional settings, see e.g., [6,18,24,27] and the references therein. In a recent paper by Mordukhovich and Nghia [23], in the finite-dimensional setting, the Hölderness and the Lipschitzness of the local minimizers to problem Par with $I_{1}=\emptyset$ are investigated for $C^{2}$ functions $\varphi_{i}, i \in I_{2}$, under MangasarianFromowitz (MFCQ) and constant rank (CRCQ) constraints qualifications.

Let $\mathcal{H}$ be a Hilbert space and let $\mathcal{D} \subset \mathcal{G}$ be a nonempty set of a normed space $\mathcal{G}$, $p \in \mathcal{D}$ and $v \in \mathcal{H}$. We consider the norm topology induced on $\mathcal{D}$ by the topology of space $\mathcal{G}$, i.e., $U$ is an open set in $\mathcal{D}$ if $U=\mathcal{D} \cap U^{\prime}$, where $U^{\prime}$ is open in $\mathcal{G}$ (see e.g. [11]).

We consider the following parametric optimization problem

$$
\begin{aligned}
& \min _{x \in \mathcal{H}} \frac{1}{2}\|x-v\|^{2}, \\
& \text { subject to } x \in C(p):=\left\{\begin{array}{l|l}
x \in \mathcal{H} & \begin{array}{l}
\left\langle x \mid g_{i}(p)\right\rangle=f_{i}(p), i \in I_{1}, \\
\left\langle x \mid g_{i}(p)\right\rangle \leq f_{i}(p), i \in I_{2}
\end{array}
\end{array}\right\},
\end{aligned}
$$

where $f_{i}: \mathcal{D} \rightarrow \mathbb{R}, g_{i}: \mathcal{D} \rightarrow \mathcal{H}, i \in I_{1} \cup I_{2} \neq \emptyset, I_{1}=\varnothing \vee\{1, \ldots, q\}$, $I_{2}=\emptyset \vee\{q+1, \ldots, m\}$ are locally Lipschitz on $\mathcal{D}$.

When $C(p) \neq \varnothing$ for $p \in \mathcal{D}$, problem $(\mathrm{M}(v, p))$ is uniquely solvable for any $v \in \mathcal{H}$ and the solution $P(v, p)$ to problem $(\mathrm{M}(v, p))$ is the projection of $v$ onto $C(p)$ i.e.

$$
P(v, p):=P_{C(p)}(v) .
$$

Our aim is to prove local Lipschitzness of the projection mapping $P: \mathcal{H} \times \mathcal{D} \rightarrow \mathcal{H}$, given by (1) at an arbitrary fixed $(\bar{v}, \bar{p})$. The following example shows that even if the functions $g_{i}: \mathcal{D} \rightarrow \mathcal{H}, i \in I_{1} \cup I_{2}$ are globally Lipschitz, the projection onto $C(p), p \in \mathcal{D}$, given by (3), may not be continuous (in the strong topology). For other examples see e.g. [31]. 
Example 1 Let $p \in \mathbb{R}, \bar{p}=0, \bar{v}=(-1,-1)$ and

$$
C(p)=\left\{\begin{array}{l|l}
x \in \mathbb{R}^{2} & \begin{array}{l}
\langle x \mid(-1+p, 0)\rangle \leq 0 \\
\langle x \mid(0, p)\rangle \leq 0
\end{array}
\end{array}\right\} .
$$

The projection of $v=\left(v_{1}, v_{2}\right)$ from a neighbourhood of $\bar{v}$ onto $C(p)$, for $p$ close to $\bar{p}$ is equal to

$$
P(p, v)=\left\{\begin{array}{l}
(0,0) \text { if }-1<p<0 \\
\left(0, v_{2}\right) \text { if } 1>p \geq 0
\end{array} .\right.
$$

Hence, $P(\cdot, \cdot)$ is not continuous at $(\bar{p}, \bar{v})$.

Our analysis is based on a recent results of [25] concerning Lipschitzness (and Hölderness) of solutions to a class of parametric variational inclusions.

Essential part of our considerations is based on the relaxed constant rank constraint qualification (RCRCQ) introduced in [19] and investigated in [3,17,20]. According to our knowledge, no result is known in the literature, in which this particular constraint qualification condition is used in the context of stability of solutions to parametric problems (Par) with $I_{1} \neq \emptyset$. Moreover, we assume only local Lipschitzness of the right-hand side functions $f_{i}, i \in I_{1} \cup I_{2}$ and left-hand side functions $g_{i}, i \in I_{1} \cup I_{2}$.

Observe, that, in general, the existing continuity-type results for solutions of problem (Par) are representation-dependent in the sense that e.g. MFCQ condition is representation-dependent (see Example 2). Observe that RCRCQ (Definition 2) is also representation-dependent. We take this fact into account by introducing the concept of equivalent representation (Definition 4) and the concept of equivalent stable representation (Definition 5). In Theorem 5 we show that the under assumption (H1) the existence of a suitable equivalent representation is necessary for the continuity of projections onto sets $C(p), p \in \mathcal{D}$, given by (3).

The organization of the paper is as follows. In Theorem 1 of Sect. 2 we recall Theorem 6.5 of [25] in the form which corresponds to our settings. Theorem 1 provides sufficient and necessary conditions for the estimate (25) which is stronger than local Lipschitzness of projection $P(\cdot, \cdot)$ (see (II) of Theorem 1). For convenience of the reader we provide the proof of the sufficiency part of Theorem 1. In Sect. 3 we recall the relaxed constant rank qualification (RCRCQ) and the results concerning Lipschitzlikeness of parametrized constrained sets $C(p), p \in \mathcal{D}$, given by (3). In Sect. 4 we investigate Lagrange multipliers of problem $(\mathrm{M}(v, p))$ under RCRCQ. In Sect. 5 we introduce the concept of equivalent stable representation of sets $C(p), p \in \mathcal{D}$, given by (3). Main results of this section are Corollary 1 and Theorem 5 . Section 6 contains the main result of the present paper (Theorem 6) together with a number of corollaries referring to several particular cases of problem $(\mathrm{M}(v, p))$. Section 7 concludes. 


\section{Underlying Facts}

In the Hilbert space setting, $P(v, p)$ given by (1) is characterized as solution to the parametric variational inequality

$$
\text { find } x \in \mathcal{H} \text { s.t } \quad v \in x+N(x ; C(p)) \text {, }
$$

where $N(x ; C(p))$ stands for the normal cone (in the sense of convex analysis) to the set $C(p)$ at $x \in C(p)$ i.e.,

$$
N(x ; C(p)):=\{h \in \mathcal{H} \mid\langle h \mid y-x\rangle \leq 0, \forall y \in C(p)\} .
$$

Equivalently,

$$
\langle v-P(v, p), y-p(v, p)\rangle \leq 0 \quad \forall y \in C(p)
$$

which is the classical characterisation of projection in Hilbert spaces.

Local Lipschitzness of solutions to general parametric variational inequalities has been recently investigated by Mordukhovich, Nghia and Pham in [25, Theorem 6.5].

The formulation of Theorem 6.5 of [25] cast to our problem is given in Theorem 1.

From the point of view of applications it is also interesting to investigate the particular case of problem $(\mathrm{M}(v, p))$ with $v \equiv \bar{v}$, i.e.,

$$
\begin{aligned}
& \min _{x \in \mathcal{H}} \frac{1}{2}\|x-\bar{v}\|^{2}, \\
& \text { subject to } x \in C(p):=\left\{\begin{array}{l|l}
x \in \mathcal{H} & \begin{array}{l}
\left\langle x \mid g_{i}(p)\right\rangle=f_{i}(p), i \in I_{1}, \\
\left\langle x \mid g_{i}(p)\right\rangle \leq f_{i}(p), i \in I_{2}
\end{array}
\end{array}\right\},
\end{aligned}
$$

where $\bar{v} \in \mathcal{H}$, i.e. the problem $(\mathrm{M}(p))$ does not depend on parameter $v$.

When $g_{i}(p) \equiv g_{i} \in \mathcal{H}, i \in I_{1} \cup I_{2}$, the sets $C(p)$ take the form

$$
\hat{C}(p):=\left\{\begin{array}{l|l}
x \in \mathcal{H} & \begin{array}{l}
\left\langle x \mid g_{i}\right\rangle=f_{i}(p), i \in I_{1}, \\
\left\langle x \mid g_{i}\right\rangle \leq f_{i}(p), i \in I_{2}
\end{array}
\end{array}\right\}
$$

and the stability of the respective variational system $(\mathrm{PV}(v, p))$ has been investigated in [30].

For any multifunction $\mathcal{F}: X \rightrightarrows Y$ its domain and graph are defined as

$$
\begin{aligned}
\operatorname{dom} \mathcal{F} & :=\{u \in X \mid \mathcal{F}(u) \neq \emptyset\}, \\
\operatorname{gph} \mathcal{F} & :=\{(u, y) \in X \times Y \mid y \in F(u)\} .
\end{aligned}
$$

An important assumption of Theorem 1, is Lipschitz-like behaviour of the multifunction $\mathcal{C}: \mathcal{P} \rightarrow \mathcal{H}, \mathcal{C}(p):=C(p)$. Below we recall this concept.

Definition 1 [21, Definition 1.40] Let $X, Y$ be normed spaces. Let $\mathcal{F}: X \rightrightarrows Y$ be a multifunction with dom $\mathcal{F} \neq \emptyset$. Given $(\bar{u}, \bar{y}) \in \operatorname{gph} \mathcal{F}$, we say that $\mathcal{F}$ is Lipschitz-like 
(pseudo-Lipschitz, has the Aubin property) around $(\bar{u}, \bar{y})$ with modulus $\ell \geq 0$ if there are neighbourhoods $U(\bar{u})$ of $\bar{u}$ and $V(\bar{y})$ of $\bar{y}$ such that

$$
\mathcal{F}\left(u_{1}\right) \cap V(\bar{y}) \subset \mathcal{F}\left(u_{2}\right)+\ell\left\|u_{1}-u_{2}\right\| B(0,1) \text { for all } u_{1}, u_{2} \in U(\bar{u}),
$$

where $B(0,1)$ is the open unit ball in $Y$.

This property is crucial in investigation of parametric problems, e.g., in variational systems [12], critical point set [14,26]. It is extensively studied in recent monographs $[9,21,22,28]$.

Let $\mathbb{C}: \mathcal{D} \rightrightarrows \mathcal{H}$ be a set-valued mapping defined as $\mathbb{C}(p):=C(p)$,

$$
C(p)=\left\{\begin{array}{l|l}
x \in \mathcal{H} & \begin{array}{l}
\left\langle x \mid g_{i}(p)\right\rangle=f_{i}(p), i \in I_{1}, \\
\left\langle x \mid g_{i}(p)\right\rangle \leq f_{i}(p), i \in I_{2}
\end{array}
\end{array}\right\},
$$

where $f_{i}: \mathcal{D} \rightarrow \mathbb{R}, g_{i}: \mathcal{D} \rightarrow \mathcal{H}, i \in I_{1} \cup I_{2} \neq \emptyset, I_{1}=\emptyset \vee\{1, \ldots, q\}$, $I_{2}=\emptyset \vee\{q+1, \ldots, m\}$ are locally Lipschitz on $\mathcal{D}$. The set $C(p)$ is the feasible solution set of problem $(\mathrm{M}(v, p))$.

In view of [25, Example 6.4], the fact [25, Lemma 6.2] applied to problem $(\mathrm{PV}(v, p))$ takes the following form.

Proposition 1 [25, Lemma 6.2] Let $\bar{x}=P(\bar{v}, \bar{p}), \bar{v} \in \mathcal{H}, \bar{p} \in \mathcal{D}$. If $\mathbb{C}$ is Lipschitz-like around $(\bar{p}, \bar{x})$, then there exist constants $\kappa_{0}, \ell^{0}>0$ and neighbourhoods $W(\bar{v}), U(\bar{p})$ that the estimate

$$
\left\|\left(v_{1}-v_{2}\right)-2 \kappa_{0}\left[P\left(v_{1}, p_{1}\right)-P\left(v_{2}, p_{2}\right)\right]\right\| \leq\left\|v_{1}-v_{2}\right\|+\ell^{0}\left\|p_{1}-p_{2}\right\|^{1 / 2}
$$

holds for all $\left(v_{1}, p_{1}\right),\left(v_{2}, p_{2}\right) \in W(\bar{v}) \times U(\bar{p})$.

Let us note, that in view of Lemma 6.2 of [25], we have $\kappa_{0}=1-\lambda r$, where in our case $\lambda=1$ and $r=0$ (Lemma 5.2 of [25] remain true for $\mathcal{R}=r=0$ ), i.e., (4) takes the form

$$
\left\|\left(v_{1}-v_{2}\right)-2\left[P\left(v_{1}, p_{1}\right)-P\left(v_{2}, p_{2}\right)\right]\right\| \leq\left\|v_{1}-v_{2}\right\|+\ell^{0}\left\|p_{1}-p_{2}\right\|^{1 / 2} .
$$

For problem $(\mathrm{M}(v, p))$, or the equivalent problem $(\mathrm{PV}(v, p))$ considered in the present paper, Theorem 6.5 of [25] takes the following form.

Theorem 1 [25, Theorem 6.5] Let $\bar{p} \in \mathcal{D}, \bar{v} \in \mathcal{H}$ and $\bar{x}=P(\bar{v}, \bar{p})$. Suppose that

(A) $\mathbb{C}$ is Lipschitz-like around $(\bar{p}, \bar{x})$.

The following conditions are equivalent.

(I) The graphical subdifferential mapping $G r: \mathcal{D} \rightrightarrows \mathcal{H} \times \mathcal{H}$ defined as

$$
G r(p):=\left\{\left(x, x^{\prime}\right) \mid x \in C(p), x^{\prime} \in N(x ; C(p))\right\}=\operatorname{gph} N(\cdot ; C(p))
$$

is Lipschitz-like around $(\bar{p}, \bar{x}, \bar{v}-\bar{x})$. 
(II) There exist neighbourhoods $W(\bar{v}), U(\bar{p})$ such that the estimate

$$
\left\|\left(v_{1}-v_{2}\right)-2\left[P\left(v_{1}, p_{1}\right)-P\left(v_{2}, p_{2}\right)\right]\right\| \leq\left\|v_{1}-v_{2}\right\|+\ell^{0}\left\|p_{1}-p_{2}\right\|
$$

holds for all $\left(v_{1}, p_{1}\right),\left(v_{2}, p_{2}\right) \in W(\bar{v}) \times U(\bar{p})$ with some positive constant $\ell^{0}$. 1.

For convenience of the Reader, we provide a direct proof of (I) $\Longrightarrow$ (II) of Theorem

Proof By Proposition 1, there exist constant $\ell^{0}>0$ and neighbourhoods $V(\bar{v}), Q(\bar{p})$ such that the estimate

$$
\left\|\left(v_{1}-v_{2}\right)-2\left[P\left(v_{1}, p_{1}\right)-P\left(v_{2}, p_{2}\right)\right]\right\| \leq\left\|v_{1}-v_{2}\right\|+\ell^{0}\left\|p_{1}-p_{2}\right\|^{1 / 2}
$$

holds for all $\left(v_{1}, p_{1}\right),\left(v_{2}, p_{2}\right) \in V(\bar{v}) \times Q(\bar{p})$. Moreover, by (I), there exist neighbourhoods $Q_{1}(\bar{p}) \subset Q(\bar{p}), U_{1}(\bar{x}), V_{1}(\bar{v}-\bar{x})$ for which $v+u \in V(\bar{v})$ whenever $(u, p, v) \in U_{1}(\bar{x}) \times Q_{1}(\bar{p}) \times V_{1}(\bar{v}-\bar{x})$ such that

$$
\operatorname{Gr}\left(p_{1}\right) \cap\left[U_{1}(\bar{x}) \times V_{1}(\bar{v}-\bar{x})\right] \subset \operatorname{Gr}\left(p_{2}\right)+\ell_{G r}\left\|p_{1}-p_{2}\right\| B(0,1)
$$

holds for all $p_{1}, p_{2} \in Q_{1}(\bar{p})$, where $\ell_{G r}>0$ is a constant (here $B(0,1)$ is the unit ball in $\mathcal{H} \times \mathcal{H})$. By $(5)$, we have

$$
\begin{aligned}
\left\|v_{1}-v_{2}\right\|+\ell^{0}\left\|p_{1}-p_{2}\right\|^{1 / 2} & \geq\left\|\left(v_{1}-v_{2}\right)-2\left[P\left(v_{1}, p_{1}\right)-P\left(v_{2}, p_{2}\right)\right]\right\| \\
& \geq\left|2\left\|P\left(v_{1}, p_{1}\right)-P\left(v_{2}, p_{2}\right)\right\|-\left\|v_{1}-v_{2}\right\|\right| \\
& \geq 2\left\|P\left(v_{1}, p_{1}\right)-P\left(v_{2}, p_{2}\right)\right\|-\left\|v_{1}-v_{2}\right\| .
\end{aligned}
$$

Hence

$$
\left\|P\left(v_{1}, p_{1}\right)-P\left(v_{2}, p_{2}\right)\right\| \leq\left\|v_{1}-v_{2}\right\|+\frac{\ell^{0}}{2}\left\|p_{1}-p_{2}\right\|^{1 / 2} .
$$

There exist neighbourhoods $U_{2}(\bar{x}), Q_{2}(\bar{p}), V_{2}(\bar{v})$, such that $U_{2}(\bar{x}) \times Q_{2}(\bar{p}) \times V_{2}(\bar{v}) \subset$ $U_{1}(\bar{x}) \times Q_{1}(\bar{p}) \times V(\bar{v})$ and $P\left(V_{2}(\bar{x}), Q_{2}(\bar{p})\right) \subset U_{2}(\bar{x})$, and

$$
v-u=v-\bar{v}-(u-\bar{x})+\bar{v}-\bar{x} \subset V_{1}(\bar{v}-\bar{x})
$$

for all $(u, p, v) \in U_{2}(\bar{x}) \times Q_{2}(\bar{p}) \times V_{2}(\bar{v})$. Now pick $\left(v_{1}, p_{1}\right),\left(v_{2}, p_{2}\right) \in V_{2}(\bar{v}) \times$ $Q_{2}(\bar{p})$ and define $u_{1}:=P\left(v_{1}, p_{1}\right) \in U_{2}(\bar{x})$ and $u_{2}:=P\left(v_{2}, p_{2}\right) \in U_{2}(\bar{x})$. Therefore, we have $v_{1}^{\prime}:=v_{1}-u_{1} \in N\left(u_{1} ; C\left(p_{1}\right)\right) \cap V_{1}(\bar{v}-\bar{x})$, i.e., $\left(u_{1}, v_{1}^{\prime}\right) \in G r\left(p_{1}\right) \cap\left(U_{1}(\bar{x}) \times\right.$ $\left.V_{1}(\bar{v}-\bar{x})\right)$. By $(8)$, there is $(u, v) \in G r\left(p_{2}\right)$ satisfying

$$
\left\|u-u_{1}\right\|+\left\|v-v_{1}^{\prime}\right\| \leq \ell_{G r}\left\|p_{1}-p_{2}\right\| .
$$

Define $v^{\prime}:=u+v \in u+N\left(u ; C\left(p_{2}\right)\right)$, i.e., $u=P\left(v^{\prime}, C\left(p_{2}\right)\right)$. Then

$$
\left\|v^{\prime}-v_{1}\right\|=\left\|u+v-u_{1}-v_{1}^{\prime}\right\| \leq\left\|v-v_{1}^{\prime}\right\|+\left\|u-u_{1}\right\| \leq \ell_{G r}\left\|p_{1}-p_{2}\right\| .
$$


Hence, $V^{\prime}(\bar{v}) \subset V(\bar{v})$ by choosing $Q_{2}(\bar{p})$ sufficiently small. By (7), for pairs $\left(v^{\prime}, p_{2}\right)$ and $\left(v_{2}, p_{2}\right)$ we have

$$
\left\|\left(v^{\prime}-v_{2}\right)-2\left(u-u_{2}\right)\right\| \leq\left\|v^{\prime}-v_{2}\right\| .
$$

Hence, for any $\left(v_{1}, p_{1}\right),\left(v_{2}, p_{2}\right) \in V_{2}(\bar{v}) \times Q_{2}(\bar{p})$,

$$
\begin{aligned}
\left\|\left(v_{1}-v_{2}\right)-2\left(u_{1}-u_{2}\right)\right\| & \leq\left\|\left(v^{\prime}-v_{2}\right)-2\left(u-u_{2}\right)\right\|+\left\|v^{\prime}-v_{1}\right\|+2\left\|u_{1}-u\right\| \\
& \leq\left\|v^{\prime}-v_{2}\right\|+\left\|v^{\prime}-v_{1}\right\|+2\left\|u_{1}-u\right\| \\
& \leq\left\|v_{1}-v_{2}\right\|+\left\|v^{\prime}-v_{1}\right\|+\left\|v^{\prime}-v_{1}\right\|+2\left\|u_{1}-u\right\| \\
& \leq\left\|v_{1}-v_{2}\right\|+4 \ell_{G r}\left\|p_{1}-p_{2}\right\| .
\end{aligned}
$$

Remark 1 It follows from the proof that under the assumptions of Theorem 1 and condition (I), the estimate in (II) holds with constant $\ell^{0}=4 \ell_{G r}$.

Remark 2 Clearly, dom $G r=\{p \in \mathcal{G} \mid G r(p) \neq \emptyset\}=\operatorname{dom} \mathbb{C}$ and

$$
G r(p)=\{(x, 0) \mid x \in \operatorname{int} C(p)\} \cup\left\{\left(x, x^{\prime}\right) \mid x \in \operatorname{bd} C(p), x^{\prime} \in N(x ; C(p)) \neq\{0\}\right\}
$$

for $p \in \operatorname{dom} G r$. Consequently, by taking $\bar{p} \in \operatorname{dom} \mathbb{C}, 0 \neq \bar{v}-\bar{x} \in N(\bar{x} ; C(\bar{p}))$ and a neighbourhood $V(0)$ in $\mathcal{H}$ such that $0 \notin \bar{v}-\bar{x}+V(0)$

$$
x \in(\bar{x}+V(0)) \cap C(p) \wedge x^{\prime} \in(\bar{v}-\bar{x}+V(0)) \cap N(x ; C(p)) \Rightarrow x \in \operatorname{bd} C(p)(9)
$$

for $p$ close to $\bar{p}$.

In view of Theorem 1 to prove (II) we need to show (I) and the condition (A) . Condition (A) for $\mathcal{C}$ given by (2) was investigated in details in [3,5] and it is discussed in Sect. 3. Condition (I) is proved in Proposition 9 in Sect. 6 with the help of a number of propositions proved in Sect. 4.

In the sequel we make an extensive use of the lower Kuratowski limit (PainlevéKuratowski inner/lower limit) for a multifunction $\mathcal{F}: \mathcal{D} \rightrightarrows \mathcal{H}$ at $\bar{p}$ defined as

$$
\liminf _{p \rightarrow \bar{p}, p \in \mathcal{D}} \mathcal{F}(p):=\left\{y \in \mathcal{H} \mid \forall p_{k} \rightarrow \bar{p}, p_{k} \in \mathcal{D}, \exists y_{k} \in \mathcal{F}\left(p_{k}\right) \quad y_{k} \rightarrow y\right\}
$$

Equivalently, $\bar{x} \in \liminf _{p \rightarrow \bar{p}, p \in \mathcal{D}} \mathcal{F}(p)$ if for every neighbourhood $V(\bar{x})$ of $\bar{x}$ there exists a neighbourhood $U(\bar{p})$ of $\bar{p}$ such that $V(\bar{x}) \cap \mathcal{F}(p) \neq \emptyset$ for $p \in U(\bar{p})$.

The following condition related to the lower Kuratowski limit is necessary for the continuity of the projection mapping $P$.

Proposition 2 Let $\bar{p} \in \mathcal{D}$ and $\bar{v} \in \mathcal{H}$. If the mapping $P: \mathcal{H} \times \mathcal{D} \rightarrow \mathcal{H}$ given by (1) is continuous at $(\bar{v}, \bar{p}) \in \mathcal{H} \times \mathcal{D}$ with $\bar{x}:=P(\bar{v}, \bar{p}) \in C(\bar{p})$, then $\bar{x} \in \liminf _{p \rightarrow \bar{p}, p \in \mathcal{D}} \mathbb{C}(p)$. 
Proof Suppose, by contradiction, that $\bar{x} \notin \liminf _{p \rightarrow \bar{p}, p \in \mathcal{D}} \mathbb{C}(p)$. By definition, there exists a neighbourhood $V(\bar{x})$ of $\bar{x}$ such that in every neighbourhood $U(\bar{p})$ of $\bar{p}$ there exists $p_{U} \in U(\bar{p})$ satisfying

$$
V(\bar{x}) \cap C\left(p_{U}\right)=\emptyset
$$

In consequence, $P\left(\bar{v}, p_{U}\right) \notin V(\bar{x})$, which contradicts the continuity of $P$ at $(\bar{v}, \bar{p}) . \square$

\section{RCRCQ and Lipschitz-Likeness of the Set-Valued Mapping $\mathbb{C}$}

In this section we discuss Lipschitz-likeness of set-valued mapping $\mathbb{C}: \mathcal{D} \rightrightarrows \mathcal{H}$ defined by (3).

A crucial assumption of Theorem 1 is the Lipschitz-likeness of the set-valued mapping $\mathcal{C}$ given by (3). In this section we present recent results of [4] where the the Lipschiz-likeness of $\mathcal{C}$ has been investigated with the help of the relaxed constant rank constaint qualification (RCRCQ).

For any $(p, x) \in \mathcal{D} \times \mathcal{H}$, let $I_{p}(x):=\left\{i \in I_{1} \cup I_{2} \mid\left\langle x \mid g_{i}(p)\right\rangle=f_{i}(p)\right\}$ denote the active index set for $p \in \mathcal{D}$ at $x \in \mathcal{H}$.

In our main results (Proposition 5, Theorem 6) we use the relaxed constant rank constraint qualification as defined in [4, Definition 4].

Definition 2 The relaxed constant rank constraint qualification (RCRCQ) for multifunction $\mathbb{C}$ is satisfied at $(\bar{p}, \bar{x}), \bar{x} \in C(\bar{p})$, given by (3), if there exists a neighbourhood $U(\bar{p})$ of $\bar{p}$ such that, for any index set $J, I_{1} \subset J \subset I_{\bar{p}}(\bar{x})$, for every $p \in U(\bar{p})$ the system of vectors $\left\{g_{i}(p), i \in J\right\}$ has constant rank, i.e.,

$$
\operatorname{rank}\left\{g_{i}(p), i \in J\right\}=\operatorname{rank}\left\{g_{i}(\bar{p}), i \in J\right\} \text { for all } p \in U(\bar{p})
$$

Due to the linearity with respect to $x$ of functions defining the set $C(p)$, condition (10) does not depend upon $x$ from a neighbourhood of $\bar{x}$. It is worth to mention that RCRCQ does not imply MFCQ, nor MFCQ imply RCRCQ (see e.g. [15])

Proposition 3 says that in a neighbourhood of $\bar{p} \in \mathcal{D}$ we can equivalently represent the set $C(p)$, given by (3), in such way that the normal vectors of equality constraints are linearly independent. A finite-dimensional analogue of Proposition 3 has been established in [17, Lemma 2.2].

Proposition 3 [4, Proposition 11] Let $\bar{p} \in \mathcal{D}$. Assume RCRCQ holds at $(\bar{p}, \bar{x}), \bar{x} \in$ $C(\bar{p})$ for multifunction $\mathbb{C}$ and $C(p) \neq \emptyset$ for $p \in U_{0}(\bar{p})$. There exists a neighbourhood $U(\bar{p})$ such that for all $p \in U(\bar{p})$

$$
\begin{aligned}
& \left\{x \mid\left\langle x \mid g_{i}(p)\right\rangle=f_{i}(p), i \in I_{1},\left\langle x \mid g_{i}(p)\right\rangle \leq f_{i}(p), i \in I_{2}\right\} \\
& \quad=\left\{x \mid\left\langle x \mid g_{i}(p)\right\rangle=f_{i}(p), i \in I_{1}^{\prime},\left\langle x \mid g_{i}(p)\right\rangle \leq f_{i}(p), i \in I_{2}\right\},
\end{aligned}
$$

where $I_{1}^{\prime} \subset I_{1},\left|I_{1}^{\prime}\right|=\operatorname{rank}\left\{g_{i}(\bar{p}), i \in I_{1}\right\}$ and $g_{i}(p), i \in I_{1}^{\prime}$ are linearly independent. 
In view of Proposition 3, in the sequel we assume that for any $\bar{p} \in \mathcal{D}, g_{i}(p), i \in I_{1}$ are linearly independent in some neighbourhood $U(\bar{p})$.

Remark 3 Let us note that for the set-valued mapping $\hat{\mathbb{C}}: \mathcal{D} \rightrightarrows \mathcal{H}, \hat{\mathbb{C}}(p):=\hat{C}(p)$, with $\hat{C}(p)$ defined by (2), the relaxed constant rank constraint qualification condition $\mathrm{RCRCQ}$ is satisfied at any $(p, x) \in \mathrm{gph} \hat{\mathbb{C}}$. In the case of absence of equality constraints in (3) the condition RCRCQ is equivalent to constant rank constraint qualification (CRCQ) (see [1,13,16]) which has been already used in [23] in proving Lipschitzness of projections.

The following theorem has been proved in [3].

Theorem 2 [4, Theorem 9] Let $\mathcal{H}$ be a Hilbert space, $\mathcal{D} \subset \mathcal{G}$ be a subset of a normed space $\mathcal{G}$ and let $\mathbb{C}: \mathcal{D} \rightrightarrows \mathcal{H}$ be given by (3). Assume RCRCQ is satisfied at $(\bar{p}, \bar{x}) \in$ gph $\mathbb{C}$ and $\bar{x} \in \liminf _{p \rightarrow \bar{p}, p \in \mathcal{D}} \mathbb{C}(p)$. Then $\mathbb{C}$ is Lipschitz-like at $(\bar{p}, \bar{x})$.

By applying Proposition 1 and Theorem 2, we immediately obtain the following Hölder type estimate for solutions to problem $(\mathrm{M}(v, p))$.

Theorem 3 Let $\mathcal{H}$ be a Hilbert space, $\mathcal{D} \subset \mathcal{G}$ be a subset of a normed space $\mathcal{G}$ and let $\mathbb{C}: \mathcal{D} \rightrightarrows \mathcal{H}$ be given by (3). Assume RCRCQ is satisfied at a point $(\bar{p}, \bar{x}) \in$ gph $\mathbb{C}$ and $\bar{x} \in \liminf _{p \rightarrow \bar{p}, p \in \mathcal{D}} \mathbb{C}(p)$. Then there exist constant $\ell^{0}>0$ and neighbourhoods $W(\bar{v})$, $U(\bar{p})$ that the estimate

$$
\left\|\left(v_{1}-v_{2}\right)-2\left[P\left(v_{1}, p_{1}\right)-P\left(v_{2}, p_{2}\right)\right]\right\| \leq\left\|v_{1}-v_{2}\right\|+\ell^{0}\left\|p_{1}-p_{2}\right\|^{1 / 2}
$$

holds for all $\left(v_{1}, p_{1}\right),\left(v_{2}, p_{2}\right) \in W(\bar{v}) \times U(\bar{p})$.

In view of Remark 3, the following result is an immediate consequence of Theorem 2.

Theorem 4 Let $\mathcal{H}$ be a Hilbert space, $\mathcal{D} \subset \mathcal{G}$ be a subset of a normed space $\mathcal{G}$ and let $\hat{\mathbb{C}}: \mathcal{D} \rightrightarrows \mathcal{H}, \hat{\mathbb{C}}(p):=\hat{C}(p)$, with $\hat{C}(p)$ given by $(2)$. Assume that $(\bar{p}, \bar{x}) \in$ gph $\hat{\mathbb{C}}$ and $\bar{x} \in \liminf _{p \rightarrow \bar{p}, p \in \mathcal{D}} \hat{\mathbb{C}}(p)$. Then $\hat{\mathbb{C}}$ is Lipschitz-like at $(\bar{p}, \bar{x})$.

The Lipschitz-likeness of $\hat{\mathbb{C}}$ has already been investigated in the finite-dimensional case in [10] with the help of the Mangasarian-Fromowitz constraint qualification MFCQ.

Definition 3 We say that the Mangasarian-Fromowitz constraint qualification (MFCQ) holds for $C(\bar{p})$ at $\bar{x} \in C(\bar{p})$ if vectors $g_{i}(\bar{p}), i \in I_{1}$ are linearly independent and there exists $h \in \mathcal{H}$ such that

$$
\left\langle g_{i}(\bar{p}) \mid h\right\rangle=0 \quad i \in I_{1}, \quad\left\langle g_{i}(\bar{p}) \mid h\right\rangle<0 \quad i \in I_{\bar{p}}(\bar{x})
$$

The following fact relates the Mangasarian-Fromowitz constraint qualification MFCQ to the lower Kuratowski limit of the set-valued mapping $\overline{\mathbb{C}}: \mathcal{D} \rightrightarrows \mathcal{H}$, $\overline{\mathbb{C}}(p):=\bar{C}(p)$, with $\bar{C}(p)$ given by (3) with $I_{1}=\emptyset$, i.e. 


$$
\bar{C}(p):=\left\{x \in \mathcal{H} \mid\left\langle x \mid g_{i}(p)\right\rangle \leq f_{i}(p), i \in I_{2}\right\} .
$$

Proposition 4 If MFCQ holds for $\bar{C}(\bar{p})$ at $\bar{x} \in \bar{C}(\bar{p})$, then $\bar{x} \in \liminf _{p \rightarrow \bar{p}, p \in \mathcal{D}} \bar{C}(p)$.

Proof By MFCQ, there exists $h \in \mathcal{H}$ such that

$$
\left\langle g_{i}(\bar{p}) \mid h\right\rangle<0 \text { for } i \in I_{\bar{p}}(\bar{x}) \text {. }
$$

Let $V(\bar{x})$ be any neighbourhood of $\bar{x}$. There exists $\alpha>0$ such that

$$
\left\langle g_{i}(\bar{p}) \mid \bar{x}+\alpha h\right\rangle<f_{i}(\bar{p}) \quad i \in I_{2}
$$

and $\bar{x}+\alpha h \in V(\bar{x})$. Since the functions $g_{i}, f_{i}, i \in I_{1} \cup I_{2}$ are assumed to be locally Lipschitz at $\bar{p}$ there exists a neighbourhood $U_{i}(\bar{p})$ of $\bar{p}$ such that

$$
\begin{aligned}
& \left\langle g_{i}(p)-g_{i}(\bar{p}) \mid \bar{x}+\alpha h\right\rangle \leq \ell_{g_{i}}\|p-\bar{p}\|\|\bar{x}+\alpha \cdot h\| p \in U_{i}(\bar{p}), i \in I_{2}, \\
& f_{i}(\bar{p})-\ell_{f_{i}}\|p-\bar{p}\| \leq f_{i}(p) \quad p \in U_{i}(\bar{p}), i \in I_{2}
\end{aligned}
$$

where $\ell_{f_{i}}, \ell_{g_{i}}$ are locally Lipschitz constants of functions $f_{i}, g_{i}, i \in I_{1} \cup I_{2}$ at $\bar{p}$, respectively.

Take $\varepsilon>0, \varepsilon<f_{i}(\bar{p})-\left\langle g_{i}(\bar{p}) \mid \bar{x}+\alpha h\right\rangle>0$. By shrinking the neighbourhood $U_{i}(\bar{p}), i \in I_{2}$, we can assume that

$$
\ell_{g_{i}}\|p-\bar{p}\|\|\bar{x}+\alpha \cdot h\|+\ell_{f_{i}}\|p-\bar{p}\|<\varepsilon .
$$

Consequently, for $p \in U_{i}(\bar{p}), i \in I_{2}$ we have

$$
\left\langle g_{i}(\bar{p}) \mid \bar{x}+\alpha h\right\rangle+\ell_{g_{i}}\|p-\bar{p}\|\|\bar{x}+\alpha \cdot h\|+\ell_{f_{i}}\|p-\bar{p}\| \leq\left\langle g_{i}(\bar{p}) \mid \bar{x}+\alpha h\right\rangle+\varepsilon \leq f_{i}(\bar{p})
$$

By (11),

$$
\left\langle g_{i}(p) \mid \bar{x}+\alpha h\right\rangle \leq f_{i}(\bar{p})-\ell_{f_{i}}\|p-\bar{p}\| \leq f_{i}(p), \quad i \in I_{2} .
$$

By taking $U(\bar{p})=\bigcap_{i \in I_{2}} U_{i}(\bar{p})$, we obtain the assertion.

In view of Proposition 4, Theorem 4 is a stronger result than Theorem 4.1 of [10] when applied to the linear case.

The following example shows that MFCQ is not a necessary condition for Lipschitz continuity of projection of $v$ onto $C(p), p \in \mathcal{D}$, given in (3).

Example 2 Let $p \in B((0,0), 1) \subset \mathbb{R}^{2}, \bar{p}=(0,0), \bar{v}=(1,1)$ and

$$
C(p)=\left\{\begin{array}{l|l}
x \in \mathbb{R}^{2} & \begin{array}{l}
\langle x \mid(1,0)-p\rangle-\langle p \mid(1,0)-p\rangle \leq 0 \\
\langle x \mid(0,1)-p\rangle-\langle p \mid(0,1)-p\rangle \leq 0 \\
\langle x \mid(-1,-1)-p\rangle-\langle p \mid(-1,-1)-p\rangle \leq 0
\end{array}
\end{array}\right\}
$$


Then for all $p \in B((0,0), 1)$ we have $C(p)=\{p\}$. Hence $P(v, p)=p$ for $p \in$ $B((0,0), 1)$ and for any $v \in \mathbb{R}^{2}$. Hence, $P(\cdot, \cdot)$ is locally Lipschitz in a neighbourhood of $(\bar{v}, \bar{p})$ and MFCQ is not satisfied at $P(\bar{v}, \bar{p})$.

\section{RCRCQ and Lagrange Multipliers}

In our investigations of Lipschitzness of the projection $P(\bar{v}, \bar{p})$ we are making an extensive use of representations of elements of normal cones (optimality conditions) and the behaviour of these representations in a neighbourhood of a given parameter $\bar{p}$. This requires the detailed investigation of the behaviour of the Lagrange multipliers around $\bar{p}$ (Proposition 5 and Proposition 7).

In this section we investigate properties of Lagrange multipliers of problem $(\mathrm{M}(v, p))$ under RCRCQ condition. We start with the following elementary observation.

Remark 4 Let $\bar{p} \in \mathcal{D}, \bar{x} \in C(\bar{p})$, where $\mathbb{C}$ is given by (3). By the continuity of $f_{i}, g_{i}$, $i \in I_{1} \cup I_{2}$, at $\bar{p}$ and the continuity of the inner product, there exist a neighbourhood $U(\bar{p})$ and a neighbourhood $V(0)$ of $0 \in \mathcal{H}$, such that

$$
\begin{aligned}
& \left\langle x \mid g_{i}(p)\right\rangle<f_{i}(p) \quad i \in I_{2} \backslash I_{\bar{p}}(\bar{x}) \\
& \left\langle x \mid g_{i}(p)\right\rangle \leq f_{i}(p) \quad i \in I_{\bar{p}}(\bar{x})
\end{aligned}
$$

for all $p \in U_{2}(\bar{p}), x \in(\bar{x}+V(0)) \cap C(p)$. Hence, $I_{p}(x) \subset I_{\bar{p}}(\bar{x})$ for $p \in U(\bar{p})$ and $x \in(\bar{x}+V(0)) \cap C(p)$.

In Proposition 5 we investigate representations of elements of $N(\bar{x} ; C(\bar{p}))$ of the form (12) below in a neighbourhood of $(\bar{p}, \bar{x}, \bar{v}-\bar{x})$, where $\bar{v}-\bar{x} \in N(\bar{x} ; C(\bar{p}))$. We prove that for all $p$ close to $\bar{p}$, for all $x \in C(p)$ close to $\bar{x}$, for all $x^{\prime} \in N(x ; C(p))$ close to $\bar{v}-\bar{x}$, there exists a representation

$$
x^{\prime}=\sum_{i \in I_{\bar{p}}(\bar{x})} \tilde{\lambda}_{i} g_{i}(p)
$$

and the function $\lambda: U(\bar{p}) \times(\bar{x}+V(0)) \times(\bar{v}-\bar{x}+V(0)) \rightarrow \mathbb{R}^{\left|I_{\bar{p}}(\bar{x})\right|}$,

$$
\lambda\left(p, x, x^{\prime}\right):= \begin{cases}\left(\tilde{\lambda}_{i}\right)_{i \in I_{\bar{p}}(\bar{x})} & \text { if }\left(p, x, x^{\prime}\right) \neq(\bar{p}, \bar{x}, \bar{v}-\bar{x}), \\ \left(\bar{\lambda}_{i}\right)_{i \in I_{\bar{p}}(\bar{x})} \text { if }\left(p, x, x^{\prime}\right)=(\bar{p}, \bar{x}, \bar{v}-\bar{x})\end{cases}
$$

is continuous at $(\bar{p}, \bar{x}, \bar{v}-\bar{x})$.

Proposition 5 Let $\bar{p} \in \mathcal{D}$. Suppose that $\bar{v} \notin C(\bar{p}), \bar{x} \in C(\bar{p}), I_{\bar{p}}(\bar{x}) \neq \emptyset .{ }^{1}$ Assume that RCRCQ holds at $\bar{p}$ (with a neighbourhood $U_{0}(\bar{p})$ ) for multifunction $\mathbb{C}$, given by

\footnotetext{
${ }^{1}$ With this assumption we limit our attention to point $\bar{x}$ which lay on the boundary of $C(\bar{p}), \bar{x} \in \operatorname{bd} C(\bar{p})$, where bd denotes the boundary of a set.
} 
(3), and $C(p) \neq \emptyset$ for $p \in U_{0}(\bar{p})$. Let

$$
\bar{v}-\bar{x}=\sum_{i \in I_{1} \cup \bar{K}} \bar{\lambda}_{i} g_{i}(\bar{p}) \text {, where } \bar{\lambda}_{i}>0, i \in \bar{K} \subset I_{\bar{p}}(\bar{x}) \cap I_{2}
$$

and $g_{i}(\bar{p}), i \in I_{1} \cup \bar{K}$ are linearly independent ${ }^{2}$. Then the following conditions hold.

(i) There exist neighbourhoods $U(\bar{p}), V(0)$ such that for any $p \in U(\bar{p})$ and any $\left(x, x^{\prime}\right) \in G r(p) \cap(\bar{x}+V(0), \bar{v}-\bar{x}+V(0))$, where $G r$ is given by (6), there exists $L \subset\left(I_{p}(x) \cap I_{2}\right) \backslash \bar{K} \subset\left(I_{\bar{p}}(\bar{x}) \cap I_{2}\right) \backslash \bar{K}^{3}$ such that the element $x^{\prime}$ can be represented as

$$
\begin{aligned}
& x^{\prime}=\sum_{i \in I_{1}} \lambda_{i} g_{i}(p)+\sum_{i \in \bar{K}} \lambda_{i} g_{i}(p)+\sum_{i \in L} \lambda_{i} g_{i}(p), \\
& \lambda_{i}>0, i \in \bar{K}, \lambda_{i} \geq 0, i \in L,
\end{aligned}
$$

where $g_{i}(p), i \in I_{1} \cup \bar{K} \cup L$ are linearly independent.

(ii) For any $\varepsilon>0$ one can choose in (i) neighbourhoods $U(\bar{p}), V(0)$ such that in the representation (13) we have

$$
\begin{aligned}
& \bar{\lambda}_{i}-\varepsilon \leq \lambda_{i} \leq \bar{\lambda}_{i}+\varepsilon \quad \forall i \in I_{1} \cup \bar{K} \\
& 0 \leq \lambda_{i} \leq \varepsilon \quad \forall i \in L
\end{aligned}
$$

Proof Since $g_{i}(\bar{p}), i \in I_{1} \cup \bar{K}$ are linearly independent and $g_{i}: \mathcal{D} \rightarrow \mathcal{H}, i \in I_{1} \cup \bar{K}$ are continuous at $\bar{p}$, by Lemma 1 (see Appendix), there exists a neighbourhood $U_{1}(\bar{p})$ such that $g_{i}(p), i \in I \cup \bar{K}, p \in U_{1}(\bar{p})$ are linearly independent.

By Remark 2 and Remark 4, there exist neighbourhoods $U_{2}(\bar{p}) \subset U_{1}(\bar{p})$ and $V_{1}(0)$, such that for all $p \in U_{2}(\bar{p})$ if $\left(x, x^{\prime}\right) \in G r(p) \cap\left(\bar{x}+V_{1}(0), \bar{v}-\bar{x}+V_{1}(0)\right)$, then $I_{p}(x) \subset I_{\bar{p}}(\bar{x}), x \in$ bd $C(p)$ and $x^{\prime} \neq 0$. Moreover, by [7, Theorem 6.40] ${ }^{4}$ we have

$$
x^{\prime}=\sum_{i \in I_{p}(x)} \lambda_{i} g_{i}(p), \quad \lambda_{i} \geq 0, i \in I_{p}(x) \cap I_{2} .
$$

Now, on the contrary suppose that the assertion of the proposition does not hold, i.e., there exist sequences $p_{n} \rightarrow \bar{p}, x_{n} \rightarrow \bar{x}, x_{n} \in C\left(p_{n}\right), x_{n}^{\prime} \rightarrow \bar{v}-\bar{x}, x_{n}^{\prime} \in N\left(x_{n} ; C\left(p_{n}\right)\right)$ such that

$$
\forall_{n \in \mathbb{N}} \quad x_{n}^{\prime} \text { can not be represented in the form (13). }
$$

\footnotetext{
2 We admit $K=\emptyset$.

3 We admit $L=\emptyset$.

${ }^{4}$ Let us note that each equality constraint in the set $C(\cdot)$ can be represented as two inequalities, namely $\langle x \mid g(p)\rangle=f_{i}(p) \Longleftrightarrow\langle x \mid g(p)\rangle \leq f_{i}(p) \wedge\langle x \mid-g(p)\rangle \leq f_{i}(p)$ for any $x \in \mathcal{H}, p \in \mathcal{D}$. 
By (14), for all $n \in \mathbb{N}$, sufficiently large, $x_{n}^{\prime}$ can be represented in the form

$$
x_{n}^{\prime}=\sum_{i \in I_{1}} \lambda_{i}^{n} g_{i}\left(p_{n}\right)+\sum_{i \in I_{p_{n}}\left(x_{n}\right) \cap I_{2}} \lambda_{i}^{n} g_{i}\left(p_{n}\right)
$$

where $\lambda_{i}^{n} \geq 0, i \in I_{p_{n}}\left(x_{n}\right) \cap I_{2}$. We can rewrite (16) as

$$
x_{n}^{\prime}=\sum_{i \in I_{1}} \lambda_{i}^{n} g_{i}\left(p_{n}\right)+\sum_{i \in I_{p_{n}}\left(x_{n}\right) \cap \bar{K}} \lambda_{i}^{n} g_{i}\left(p_{n}\right)+\sum_{i \in\left(I_{p_{n}}\left(x_{n}\right) \backslash \bar{K}\right) \cap I_{2}} \lambda_{i}^{n} g_{i}\left(p_{n}\right),
$$

and, by putting $\lambda_{i}^{n}=0$ for $i \in \bar{K} \backslash I_{p_{n}}\left(x_{n}\right), n \in \mathbb{N}$, we get

$$
x_{n}^{\prime}=\sum_{i \in I_{1}} \lambda_{i}^{n} g_{i}\left(p_{n}\right)+\sum_{i \in \bar{K}} \lambda_{i}^{n} g_{i}\left(p_{n}\right)+\sum_{i \in\left(I_{p_{n}}\left(x_{n}\right) \backslash \bar{K}\right) \cap I_{2}} \lambda_{i}^{n} g_{i}\left(p_{n}\right),
$$

where $\lambda_{i}^{n} \geq 0, i \in\left(I_{p_{n}}\left(x_{n}\right) \cap I_{2}\right) \cup \bar{K}$. By Lemma 3 (see Appendix), for all $n \in \mathbb{N}$, sufficiently large, there exist $\hat{I}_{2}^{n} \subset\left(I_{p_{n}}\left(x_{n}\right) \backslash \bar{K}\right) \cap I_{2}$ and $\tilde{\lambda}_{i}^{n}, i \in I_{1} \cup \bar{K} \cup \hat{I}_{2}^{n}, \tilde{\lambda}_{i}^{n} \in \mathbb{R}$, $i \in I_{1} \cup \bar{K}, \tilde{\lambda}_{i}^{n}>0, i \in \hat{I}_{2}^{n}$ such that

$$
x_{n}^{\prime}=\sum_{i \in I_{1}} \tilde{\lambda}_{i}^{n} g_{i}\left(p_{n}\right)+\sum_{i \in \bar{K}} \tilde{\lambda}_{i}^{n} g_{i}\left(p_{n}\right)+\sum_{i \in \hat{I}_{2}^{n}} \tilde{\lambda}_{i}^{n} g_{i}\left(p_{n}\right)
$$

where $\tilde{\lambda}_{i} \in \mathbb{R}, i \in I_{1} \cup \bar{K}, \tilde{\lambda}_{i}>0, i \in \hat{I}_{2}^{n}$ and $g_{i}\left(p_{n}\right), i \in I_{1} \cup \bar{K} \cup \hat{I}_{2}^{n}$ are linearly independent.

By passing to a subsequence, if necessary, we can assume that $\hat{I}_{2}^{n}=: I_{2}^{\prime}$ and

$$
x_{n}^{\prime}=\sum_{i \in I_{1}} \tilde{\lambda}_{i}^{n} g_{i}\left(p_{n}\right)+\sum_{i \in \bar{K}} \tilde{\lambda}_{i}^{n} g_{i}\left(p_{n}\right)+\sum_{i \in I_{2}^{\prime}} \tilde{\lambda}_{i}^{n} g_{i}\left(p_{n}\right)
$$

where $\tilde{\lambda}_{i}^{n}, \tilde{\lambda}_{i}^{n} \in \mathbb{R}, i \in I_{1} \cup \bar{K}, \tilde{\lambda}_{i}^{n}>0, i \in I_{2}^{\prime}$ and $g_{i}\left(p_{n}\right), i \in I_{1} \cup \bar{K} \cup I_{2}^{\prime}$ are linearly independent.

By (15), it must be $\tilde{\lambda}_{i}^{n} \leq 0$ for some $i_{n} \in \bar{K}$. Passing again to the subsequence in (17), if necessary, we conclude that there exists $i \in \bar{K}$ such that $\tilde{\lambda}_{i}^{n} \leq 0$.

On the other hand, by Lemma 4 (see Appendix), we have $\tilde{\lambda}_{i}^{n} \rightarrow \bar{\lambda}_{i}>0, i \in \bar{K}$, which leads to a contradiction. This proves (i).

To prove (ii) suppose there exist $\varepsilon>0$ and a sequence $\left\{i_{n}\right\}_{n \in \mathbb{N}} \subset I \cup \bar{K} \cup I_{2}^{\prime}$, such that in the representation (17) for each $n \in \mathbb{N}$ one of the following holds:

1. $\tilde{\lambda}_{i_{n}}>\bar{\lambda}_{i}+\varepsilon$ and $i_{n} \in I_{1} \cup \bar{K}$,

2. $\tilde{\lambda}_{i_{n}}<\bar{\lambda}_{i}-\varepsilon$ and $i_{n} \in I_{1} \cup \bar{K}$,

3. $\tilde{\lambda}_{i_{n}}>\varepsilon$ and $i_{n} \in I_{2}^{\prime}$.

By taking a subsequence of $\left\{x_{n}^{\prime}\right\}_{n \in \mathbb{N}}$, if necessary, one can assume that only one of the cases 1., 2., 3. holds for all $n \in \mathbb{N}$. On the other hand, by Lemma 4 (see Appendix), 
we have $\tilde{\lambda}_{i}^{n} \rightarrow \bar{\lambda}_{i}, i \in I_{1} \cup \bar{K}$ and $\tilde{\lambda}_{i}^{n} \rightarrow 0, i \in I_{2}^{\prime}$, which leads to a contradiction. This proves (ii).

It is clear that even if an element $\bar{v}-\bar{x}$ from the normal cone $N(\bar{x} ; C(\bar{p}))$ has a unique representation as a combination of some vectors $g_{i}(\bar{p}), i \in I_{\bar{p}}(\bar{x})$, then, in a neighbourhood of $(\bar{p}, \bar{x}, \bar{v}-\bar{x})$, where $\bar{v}-\bar{x} \in N(\bar{x} ; C(\bar{p}))$, the elements $x^{\prime} \in$ $N(x ; C(p))$ may not have unique representations in terms of combinations of vectors $g_{i}(p), i \in I_{p}(x)$.

The example below illustrates the situation when the representation of $\bar{v}-\bar{x}$ is not unique.

Example 3 Let $p \in \mathbb{R}, \bar{v}=(0,1)$ and

$$
C(p)=\left\{\begin{array}{ll}
x \in \mathbb{R}^{2} & \begin{array}{l}
\langle x \mid(0,1)\rangle \leq 0 \\
\langle x-(|p|, 0) \mid(1,0)\rangle \leq 0 \\
\langle x-(-|p|, 0) \mid(-1,0)\rangle \leq 0
\end{array}
\end{array}\right\}
$$

In this case $P(\bar{v}, p)=(0,0):=\bar{x}$ for all $p \in \mathbb{R}$. However, for $\bar{p}=0$ we have $I_{\bar{p}}(\bar{x})=\{1,2,3\}$ and

$$
\bar{v}-\bar{x}=1 \cdot(0,1)+0 \cdot(1,0)=1 \cdot(0,1)+0 \cdot(-1,0)
$$

and for any $p \neq 0, x=(p, 0), x^{\prime} \in N(x ; C(p))$ we have

$$
\begin{aligned}
& x^{\prime}=\lambda_{1} \cdot(0,1)+\lambda_{2}(1,0), \lambda_{1}, \lambda_{2} \geq 0, p>0 \\
& x^{\prime}=\lambda_{1} \cdot(0,1)+\lambda_{2}(-1,0), \lambda_{1}, \lambda_{2} \geq 0, p<0 .
\end{aligned}
$$

In the proposition below we show, that under assumptions appearing in Theorem 6 we have $\operatorname{Gr}(p) \cap V(\bar{x}, \bar{v}-\bar{x}) \neq \emptyset$ for $\left(p, x, x^{\prime}\right)$ in some neighbourhood of $(\bar{p}, \bar{x}, \bar{v}-\bar{x})$.

Proposition 6 Let $\bar{p} \in \mathcal{D}, \bar{v} \notin C(\bar{p})$ and $\bar{x}=P(\bar{v}, \bar{p})$. Assume that RCRCQ holds at $(\bar{p}, \bar{x})$ (with a neighbourhood $U_{0}(\bar{p})$ ) for multifunction $\mathbb{C}$, given by $(3)$, and $\bar{x} \in$ $\liminf _{p \rightarrow \bar{p}, p \in \mathcal{D}} \mathbb{C}(p)$. Then

$$
\forall V(\bar{x}, \bar{v}-\bar{x}) \exists U(\bar{p}) \forall p \in U(\bar{p}) \quad G r(p) \cap V(\bar{x}, \bar{v}-\bar{x}) \neq \emptyset,
$$

i.e., $(\bar{x}, \bar{v}-\bar{x}) \in \liminf _{p \rightarrow \bar{p}, p \in \mathcal{D}} \operatorname{Gr}(p)$.

Proof On the contrary, suppose that there exist a neighbourhood $V(\bar{x}, \bar{v}-\bar{x})$ and a sequence $p_{n} \rightarrow \bar{p}$ such that

$$
G r\left(p_{n}\right) \cap V(\bar{x}, \bar{v}-\bar{x})=\emptyset .
$$

Without loss of generality we may assume that $p_{n} \in U_{0}(\bar{p})$. Let $x_{n}:=P\left(\bar{v}, p_{n}\right)$. Then, by Theorem 3, we have $x_{n} \rightarrow \bar{x}$. Moreover, $x_{n}^{\prime}:=\bar{v}-x_{n} \in N\left(x_{n} ; C\left(p_{n}\right)\right)$ and $x_{n}^{\prime} \rightarrow \bar{v}-\bar{x}$. Thus, $\left(x_{n}, x_{n}^{\prime}\right) \in \operatorname{Gr}\left(p_{n}\right) \cap V(\bar{x}, \bar{v}-\bar{x})$ for large $n$. 
Let us recall that the set of Lagrange multipliers associated with problem $(\mathrm{M}(v, p))$ is defined as

$$
\Lambda_{v}(p, x):=\left\{\lambda \in \mathbb{R}^{m} \mid v-x=\sum_{i \in I_{1} \cup I_{2}} \lambda_{i} g_{i}(p) \text { where, for } i \in I_{2}, \lambda_{i} \geq 0, \lambda_{i} g_{i}(p)=0\right\}
$$

and for $M>0$ let

$$
\Lambda_{v}^{M}(p, x):=\left\{\lambda \in \Lambda_{v}(p, x)\left|\sum_{i \in I_{1} \cup I_{2}}\right| \lambda_{i} \mid \leq M\right\}
$$

In proposition below we show that $\Lambda_{v}^{M}(p, P(v, p)) \neq \emptyset$ in some neighbourhood of $(\bar{p}, \bar{v})$ under RCRCQ and the Kuratowski limit conditions.

Proposition 7 Suppose that $\bar{v} \notin C(\bar{p})$. Assume that $R C R C Q$ holds at $(\bar{p}, P(\bar{v}, \bar{p}))$ (with a neighbourhood $U_{0}(\bar{p})$ ) for multifunction $\mathbb{C}$, given by $(3)$, and $P(\bar{v}, \bar{p}) \in$ $\liminf _{p \rightarrow \bar{p}, p \in \mathcal{D}} \mathbb{C}(p)$. Let the formula (12) hold, i.e.,

$$
\bar{v}-P(\bar{v}, \bar{p})=\sum_{i \in I_{1} \cup \bar{K}} \bar{\lambda}_{i} g_{i}(\bar{p}) \text {, where } \bar{\lambda}_{i}>0, i \in \bar{K} \subset I_{\bar{p}}(P(\bar{v}, \bar{p})) \cap I_{2},
$$

and $g_{i}(\bar{p}), i \in I_{1} \cup \bar{K}$ are linearly independent. There exist neighbourhoods $U_{1}(\bar{p})$, $V_{1}(0)$ and $M>0$ such that for all $p \in U_{1}(\bar{p})$ and $v \in \bar{v}+V_{1}(0)$ we have

$$
\Lambda_{v}^{M}(p, P(v, p)) \neq \varnothing
$$

Proof Let $\varepsilon>0$. By Proposition 5, there exist neighbourhoods $U(\bar{p}), V(0)$ such that for every $p \in U(\bar{p})$ and any $\left(x, x^{\prime}\right) \in G r(p) \cap(\bar{x}+V(0), \bar{v}-\bar{x}+V(0))$, there exists $L \subset\left(I_{p}(x) \cap I_{2}\right) \backslash K \subset\left(I_{\bar{p}}(\bar{x}) \cap I_{2}\right) \backslash K$ such that the formula (13) holds i.e.,

$$
\begin{aligned}
& x^{\prime}=\sum_{i \in I_{1}} \lambda_{i} g_{i}(p)+\sum_{i \in \bar{K}} \lambda_{i} g_{i}(p)+\sum_{i \in L} \lambda_{i} g_{i}(p), \\
& \lambda_{i}>0, i \in \bar{K}, \lambda_{i} \geq 0, i \in L,
\end{aligned}
$$

where $g_{i}(p) i \in I_{1} \cup \bar{K} \cup L$ are linearly independent and additionally

$$
\begin{aligned}
& \bar{\lambda}_{i}-\varepsilon \leq \lambda_{i} \leq \bar{\lambda}_{i}+\varepsilon \quad \forall i \in I_{1} \cup \bar{K}, \\
& 0 \leq \lambda_{i} \leq \varepsilon \quad \forall i \in L .
\end{aligned}
$$

Let $V_{1}(0)$ be such that $V_{1}(0) \subset \frac{1}{2} V(0)$. By the continuity of $P(\cdot, \cdot)$ at $(\bar{v}, \bar{p})$ (see Theorem 2 and Proposition 1), there exist neighbourhoods $U_{2}(\bar{p}), V_{2}(0) \subset \frac{1}{2} V(0)$ such that

$$
P(v, p) \subset P(\bar{v}, \bar{p})+V_{1}(0)
$$


for all $p \in U_{2}(\bar{p}), v \in \bar{v}+V_{2}(0)$. Hence,

$$
v-P(v, p) \in \bar{v}+V_{2}(0)-P(\bar{v}, \bar{p})+V_{1}(0) \subset \bar{v}-P(\bar{v}, \bar{p})+V(0) .
$$

Let $U_{1}(\bar{p}):=U(\bar{p}) \cap U_{2}(\bar{p})$. Then for all $p \in U_{1}(\bar{p}), v \in \bar{v}+V(0)$ there exists $L \subset I_{\bar{p}}(P(\bar{v}, \bar{p})) \cap I_{2}\left(L \subset\left(I_{p}(v) \cap I_{2}\right) \backslash K\right)$ such that

$$
\begin{aligned}
& P(v, p)-v=\sum_{i \in I_{1}} \lambda_{i} g_{i}(p)+\sum_{i \in \bar{K}} \lambda_{i} g_{i}(p)+\sum_{i \in L} \lambda_{i} g_{i}(p), \\
& \lambda_{i}>0, i \in \bar{K}, \lambda_{i} \geq 0, i \in L
\end{aligned}
$$

and (19) holds. This means that for all $p \in U_{1}(\bar{p}), v \in \bar{v}+V(0)$,

$$
\sum_{i \in I_{p}(P(v, p))}\left|\lambda_{i}\right|<\sum_{i \in I_{\bar{p}}(P(\bar{v}, \bar{p}))}\left(\left|\bar{\lambda}_{i}\right|+\varepsilon\right)=: M,
$$

i.e. (18) holds.

\section{Stable Representations}

As already noted in Example 3, a number of different index sets $\bar{K}$ could be used in (12). On the other hand, the set of those index sets $\bar{K}$ for which (12) holds is nonempty (may consists of the empty set only).

Definition 4 Let $\mathbb{C}: \mathcal{D} \rightrightarrows \mathcal{H}$, be given by (3). We say that $\mathcal{R}: \mathcal{D} \rightrightarrows \mathcal{H}$ is an equivalent representation of $\mathbb{C}$, if $R(p)=C(p)$ for all $p \in \mathcal{D}$ and $R$ is given as

$$
R(p):=\left\{\begin{array}{l|l}
x \in \mathcal{H} & \begin{array}{l}
\left\langle x \mid \tilde{g}_{i}(p)\right\rangle=\tilde{f}_{i}(p), i \in \tilde{I}_{1} \\
\left\langle x \mid \tilde{g}_{i}(p)\right\rangle \leq \tilde{f}_{i}(p), i \in \tilde{I}_{2}
\end{array}
\end{array}\right\},
$$

where $\tilde{f}_{i}: \mathcal{D} \rightarrow \mathbb{R}, \tilde{g}_{i}: \mathcal{D} \rightarrow \mathcal{H}, i \in \tilde{I}_{1} \cup \tilde{I}_{2}$ are locally Lipschitz on $\mathcal{D}$ and $\tilde{I}_{1} \cup \tilde{I}_{2}$ is a finite, nonempty set. For a given representation $\mathcal{R}$ of $\mathbb{C}$, we define $I_{p}^{\mathcal{R}}(x)=\{i \in$ $\left.\tilde{I}_{1} \cup \tilde{I}_{2} \mid\left\langle x \mid \tilde{g}_{i}(p)\right\rangle=\tilde{f}_{i}(p)\right\}, p \in \mathcal{D}, x \in \mathcal{H}$.

Observe that $N(x, C(p))=N(x, R(p))$ for all $p \in \mathcal{D}, x \in C(p)$. On the other hand the representations of elements from the normal cone may differ depending on the equivalent representation considered.

Consider any equivalent representation $\mathcal{R}$ of $\mathbb{C}$. Let $\bar{p} \in \mathcal{D}, \bar{x} \in R(\bar{p})=C(\bar{p})$, $I_{\bar{p}}^{\mathcal{R}}(\bar{x}) \neq \emptyset$ and let the following formula (c.f., formula (12)) hold

$$
\bar{v}-\bar{x}=\sum_{i \in \tilde{I}_{1} \cup \bar{K}} \bar{\lambda}_{i} \tilde{g}_{i}(\bar{p}), \bar{\lambda}_{i}>0, i \in \bar{K} \subset I_{\bar{p}}^{\mathcal{R}}(\bar{x}) \cap \tilde{I}_{2}
$$

where $\tilde{g}_{i}(\bar{p}), i \in \tilde{I}_{1} \cup \bar{K}$ are linearly independent. 
For a given representation $\mathcal{R}$, for any index set $L^{5}$ satisfying

$$
\begin{aligned}
& L \subset\left(I_{\bar{p}}^{\mathcal{R}}(\bar{x}) \cap \tilde{I}_{2}\right) \backslash \bar{K} \\
& \tilde{g}_{i}(\bar{p}), i \in \tilde{I}_{1} \cup \bar{K} \cup L, \text { linearly independent }
\end{aligned}
$$

we define multifunction $\mathcal{R}_{\bar{K}, L}: \mathcal{D} \rightrightarrows \mathcal{H}$ as $\mathcal{R}_{\bar{K}, L}(p):=R_{\bar{K}, L}(p)$,

$$
R_{\bar{K}, L}(p):=\left\{\begin{array}{l|l|l}
x \in \mathcal{H} & \begin{array}{l}
\left\langle x \mid \tilde{g}_{i}(p)\right\rangle=\tilde{f}_{i}(p), i \in \tilde{I}_{1} \cup \bar{K} \cup L, \\
\left\langle x \mid \tilde{g}_{i}(p)\right\rangle \leq \tilde{f}_{i}(p), i \in \tilde{I}_{2} \backslash(\bar{K} \cup L)
\end{array}
\end{array}\right\} .
$$

Note that $\bar{x} \in R_{\bar{K}, L}(\bar{p})$ for any index set L satisfying (21) and, in general, $R_{\bar{K}, L}(p) \neq$ $C_{\bar{K}, L}(p), p \in \mathcal{D}$.

Definition 5 Let $\bar{p} \in \mathcal{D}, \bar{v} \notin C(\bar{p})$ and $\bar{x} \in C(\bar{p})$. We say that multifunction $\mathbb{C}$ : $\mathcal{D} \rightrightarrows \mathcal{H}$, given by (3), has a stable representation (in the sense of Kuratowski limit) at $(\bar{p}, \bar{v}, \bar{x})$ if there exists an equivalent representation $\mathcal{R}$ of $\mathbb{C}$ for which there exists $\bar{K} \subset I_{\bar{p}}^{\mathcal{R}}(\bar{x}) \cap \tilde{I}_{2}$ such that (20) holds and

$$
\bar{x} \in \liminf _{p \rightarrow \bar{p}, p \in \mathcal{D}} R_{\bar{K}, L}(p) \text { for any } L \text { satisfying (21). }
$$

We say that $\mathcal{R}$ is a stable representation of $\mathbb{C}$ at $(\bar{p}, \bar{v}, \bar{x})$ if there exists $\bar{K}$ such that (20) and (22) hold.

Let us note that if multifunction $\mathbb{C}: \mathcal{D} \rightrightarrows \mathcal{H}$, given by (3), has a stable representation $\mathcal{R}$ at $(\bar{p}, \bar{v}, \bar{x})$ then $\bar{x} \in \liminf _{p \rightarrow \bar{p}, p \in \mathcal{D}} C(p)=\liminf _{p \rightarrow \bar{p}, p \in \mathcal{D}} R(p)$.

By Proposition 5 and Theorem 2, we obtain the following corollary.

Corollary 1 Let $\bar{p} \in \mathcal{D}, \bar{x} \in C(\bar{p}), I_{\bar{p}}(\bar{x}) \neq \emptyset$ and $\bar{v}-\bar{x} \in N(\bar{x} ; C(\bar{p}))$. Assume that there exists an equivalent representation $\mathcal{R}$ of $\mathbb{C}$ satisfying

- RCRCQ holds for multifunction $\mathcal{R}$ at $(\bar{p}, \bar{x})$,

- $\mathcal{R}$ is a stable representation at $(\bar{p}, \bar{v}, \bar{x})$ with some set $\bar{K} \subset I_{\bar{p}}^{\mathcal{R}}(\bar{x}) \cap \tilde{I}_{2}$.

There exists a constant $\ell>0$ such that for all $\varepsilon>0$ one can find neighbourhoods $U(\bar{p})$ and $V(0)$ satisfying

(1) for any $p \in U(\bar{p})$ and any $\left(x, x^{\prime}\right) \in G r(p) \cap(\bar{x}+V(0), \bar{v}-\bar{x}+V(0))$, it exists $\hat{L} \subset\left(I_{p}^{\mathcal{R}}(x) \cap \tilde{I}_{2}\right) \backslash \bar{K} \subset\left(I_{\bar{p}}^{\mathcal{R}}(\bar{x}) \cap \tilde{I}_{2}\right) \backslash \bar{K}$ satisfying (21) such that

$$
\begin{aligned}
& \exists \lambda_{i}, i \in \tilde{I}_{1} \cup \bar{K} \cup \hat{L}, \quad x^{\prime}=\sum_{i \in \tilde{I}_{1} \cup \bar{K} \cup \hat{L}} \lambda_{i} \tilde{g}_{i}(p), \\
& \bar{\lambda}_{i}-\varepsilon \leq \lambda_{i} \leq \bar{\lambda}_{i}+\varepsilon \quad \forall i \in \tilde{I}_{1} \cup \bar{K}, \\
& 0<\lambda_{i} \leq \varepsilon \quad \forall i \in \hat{L},
\end{aligned}
$$

\footnotetext{
5 We also admit the case $L=\emptyset$.
} 
(2) for every $L$ satisfying (21), every $p_{1}, p_{2} \in U(\bar{p})$ and every $x_{1} \in(\bar{x}+V(0)) \cap$ $R_{\bar{K}, L}\left(p_{1}\right)$ there exists $x_{2} \in R_{\bar{K}, L}\left(p_{2}\right)$ such that

$$
\left\|x_{1}-x_{2}\right\| \leq \ell\left\|p_{1}-p_{2}\right\|
$$

i.e., the set-valued mapping $\mathcal{R}_{\bar{K}, L}$ is Lipschitz-like at $(\bar{p}, \bar{x})$.

Proof Clearly, RCRCQ holds for any $\mathcal{R}_{\bar{K}, L}$ at $(\bar{p}, \bar{x})$, with $L$ satisfying (21).

By Proposition 5 applied to $\mathcal{R}$, there exist neighbourhoods $U_{1}(\bar{p}), V_{1}(0)$ such that assertion (1) holds.

By Theorem 2 applied to $\mathcal{R}$ at $(\bar{p}, \bar{x})$, for any $L$ satisfying (21), the multifunction $\mathcal{R}_{\bar{K}, L}$ is Lipschitz-like at $(\bar{p}, \bar{x})$ with neighbourhoods $U_{L}(\bar{p}), V_{L}(0)$ and constant $\ell_{L}>0$, i.e., assertion (2) holds.

The existence of neighbourhoods $U(\bar{p}), V(0)$ and constant $\ell>0$ satisfying the assertion follows from the fact that there is a finite number of sets $L$ satisfying (21). $\square$

In Theorem 5 we use the following assumption (H1).

(H1) There exist an equivalent representation $\mathcal{R}$ of $\mathbb{C}$, given by (3), with

$$
\bar{v}-P(\bar{v}, \bar{p})=\sum_{i \in \tilde{I}_{1} \cup \bar{K}} \bar{\lambda}_{i} \tilde{g}_{i}(\bar{p})
$$

where $\bar{\lambda}_{i}>0, i \in \bar{K}, \tilde{g}_{i}(\bar{p}), i \in \tilde{I}_{1} \cup \bar{K}$ are linearly independent $\left(\bar{K} \subset I_{2} \cap\right.$ $\left.I_{\bar{p}}^{\mathcal{R}}(P(\bar{v}, \bar{p}))\right)$, and neighbourhoods $U(\bar{p}), W(\bar{v})$ such that

(a) $\bar{K} \subset I_{p}^{\mathcal{R}}(P(v, p))$ for all $p \in U(\bar{p}), v \in W(\bar{v})$,

(b) for any $p_{n} \rightarrow \bar{p}$ and any $L \subset\left(\tilde{I}_{2} \cap I_{\bar{p}}^{\mathcal{R}}(P(\bar{v}, \bar{p}))\right) \backslash \bar{K}$ such that $\tilde{g}_{i}(\bar{p})$, $i \in \tilde{I}_{1} \cup \bar{K} \cup L$ are linearly independent there exist sequence $v_{n} \rightarrow \bar{v}$, such that $L \subset I_{p_{n}}^{\mathcal{R}}\left(P\left(v_{n}, p_{n}\right)\right)$ for $n$ sufficiently large.

Below we show that if an equivalent representation $\mathcal{R}$ of $\mathbb{C}$ satisfies assumption (H1), then the stability of $\mathcal{R}$ (in the sense of Definition 5) is necessary for continuity of projection operator $P$.

Theorem 5 Let $\mathbb{C}: \mathcal{D} \rightrightarrows \mathcal{H}$ be given by (3). Suppose that (H1) holds, i.e., there exists an equivalent representation $\mathcal{R}$ of $\mathbb{C}$ satisfying conditions $(a)$ and $(b)$. If projection $P: \mathcal{G} \times \mathcal{D} \rightarrow \mathcal{H}$, with $P(\cdot, \cdot)$ given by $(1)$, is continuous at $(\bar{v}, \bar{p}) \in \mathcal{H} \times \mathcal{D}$, then the representation $\mathcal{R}$ of $\mathbb{C}$ is stable at $(\bar{p}, \bar{v}, P(\bar{v}, \bar{p}))$.

Proof By contradiction suppose, that representation $\mathcal{R}$ of $\mathbb{C}$ is not stable at $(\bar{p}, \bar{v}, P(\bar{v}, \bar{p}))$, i.e, for any $\tilde{K}$ such that

$$
\bar{v}-P(\bar{v}, \bar{p})=\sum_{i \in \tilde{I}_{1} \cup \tilde{K}} \bar{\lambda}_{i} \tilde{g}_{i}(\bar{p}) \text {, where } \tilde{\lambda}_{i}>0, i \in \tilde{K} \subset I_{\bar{p}}^{\mathcal{R}}(P(\bar{v}, \bar{p})) \cap \tilde{I}_{2}
$$


and $\tilde{g}_{i}(\bar{p}), i \in \tilde{I}_{1} \cup \tilde{K}$ are linearly independent, there exists $\tilde{L}$ satisfying (21) such that $P(\bar{v}, \bar{p}) \notin \liminf _{p \rightarrow \bar{p}, p \in \mathcal{D}} R_{\tilde{K}, \tilde{L}}(p)$. In particular, (23) holds for $\tilde{K}=\bar{K}$ and for any $\tilde{L} \subset\left(I_{2} \cap I_{\bar{p}}^{\mathcal{R}}(P(\bar{v}, \bar{p}))\right) \backslash \bar{K}$ such that $g_{i}(\bar{p}), i \in \tilde{I}_{1} \cup \bar{K} \cup \tilde{L}$ are linearly independent.

By assumption that $P(\bar{v}, \bar{p}) \notin \liminf _{p \rightarrow \bar{p}, p \in \mathcal{D}} R_{\bar{K}, \tilde{L}}(p)$, there exists a neighbourhood $V(0)$ such that in every neighbourhood of $\bar{p}$ one can find element $p$ such that $(P(\bar{v}, \bar{p})+$ $V(0)) \cap R_{\bar{K}, \tilde{L}}(p)=\emptyset$, i.e., there exists a sequence $p_{n} \rightarrow \bar{p}$ such that $(P(\bar{v}, \bar{p})+$ $V(0)) \cap R_{\bar{K}, \tilde{L}}\left(p_{n}\right)=\emptyset$.

Consider a sequence $v_{n} \rightarrow \bar{v}$ satisfying condition (b) of (H1). Then

$$
v_{n}-P\left(v_{n}, p_{n}\right)=\sum_{i \in \tilde{I}_{1} \cup \bar{K} \cup \tilde{L}} \lambda_{i}^{n} \tilde{g}_{i}\left(p_{n}\right), \quad \lambda_{i}^{n} \geq 0, i \in \bar{K} \cup \tilde{L} .
$$

This formula implies that $\tilde{I}_{1} \cup \bar{K} \cup \tilde{L} \subset I_{p_{n}}^{\mathcal{R}}\left(P\left(v_{n}, p_{n}\right)\right), P\left(v_{n}, p_{n}\right) \in R_{\bar{K}, \tilde{L}}\left(p_{n}\right)$ and $v_{n}-P\left(v_{n}, p_{n}\right) \in N\left(x_{n}, R_{\tilde{L}}\left(p_{n}\right)\right)$. Thus $P\left(v_{n}, p_{n}\right)=P_{R_{\bar{K}, \tilde{L}}\left(p_{n}\right)}\left(v_{n}\right)$. Hence, $P\left(v_{n}, p_{n}\right) \notin P(\bar{v}, \bar{p})+V(0)$, which means that $P(\cdot, \cdot)$ is not continuous at $(\bar{v}, \bar{p})$.

\section{Main Results}

In this section we prove local Lipschitzness of projections onto moving closed convex sets $C(p)$ defined by (3). In view of Theorem 2 in order to apply Theorem 1 we need to investigate Lipschitz-likeness of the graphical subdifferential mapping $G r$ given by (6).

We start with the following technical fact.

Proposition 8 Let $(\bar{p}, \bar{x}, \bar{v}-\bar{x})$ be such that $\bar{x} \in C(\bar{p}), \bar{v}-\bar{x} \in N(\bar{x} ; C(\bar{p}))$, and $I_{\bar{p}}(\bar{x})=\left\{i \in I_{1} \cup I_{2} \mid\left\langle\bar{x} \mid g_{i}(\bar{p})\right\rangle=f_{i}(\bar{p})\right\} \neq \emptyset$. The following conditions are equivalent:

(i) The graphical subdifferential mapping $G r: \mathcal{D} \rightrightarrows \mathcal{H} \times \mathcal{H}$ defined as

$$
G r(p)=\left\{\left(x, x^{\prime}\right) \mid x \in C(p), x^{\prime} \in N(x ; C(p))\right\}
$$

is Lipschitz-like around $(\bar{p}, \bar{x}, \bar{v}-\bar{x})$

(ii) There exist $\ell>0$ and neighbourhoods $U(\bar{p}), V(0)$ in $\mathcal{H}$ such that

$$
\begin{aligned}
& \forall p_{1}, p_{2} \in U(\bar{p}) \\
& \forall x_{1} \in C\left(p_{1}\right) \cap(\bar{x}+V(0)), x_{1}^{\prime} \in N\left(x_{1} ; C\left(p_{1}\right)\right) \cap(\bar{v}-\bar{x}+V(0)) \\
& \exists x_{2} \in C\left(p_{2}\right), x_{2}^{\prime} \in N\left(x_{2} ; C\left(p_{2}\right)\right) \text { satisfying } \\
& \left\|x_{1}-x_{2}\right\| \leq \ell\left\|p_{1}-p_{2}\right\|,
\end{aligned}
$$

$$
\left\|x_{1}^{\prime}-x_{2}^{\prime}\right\| \leq \ell\left\|p_{1}-p_{2}\right\|
$$


Proof By (i), there exist neighbourhoods $U(\bar{p}), V(0)$ such that for every $\left(p_{1}, p_{2}\right) \in$ $U(\bar{p})$

$$
\begin{gathered}
G r\left(p_{1}\right) \cap(\bar{x}+V(0), \bar{v}-\bar{x}+V(0)) \\
\subset G r\left(p_{2}\right)+\ell\left\|p_{1}-p_{2}\right\| B(0,1),
\end{gathered}
$$

i.e., for all $\left(x_{1}, x_{1}^{\prime}\right) \in \operatorname{gph} G r\left(p_{1}\right) \cap(\bar{x}+V(0), \bar{v}-\bar{x}+V(0))$ there exists $\left(x_{2}, x_{2}^{\prime}\right) \in$ $G r\left(p_{2}\right)$ such that

$$
\left(x_{1}, x_{1}^{\prime}\right) \in\left(x_{2}, x_{2}^{\prime}\right)+\ell\left\|p_{1}-p_{2}\right\| B(0,1)
$$

where $B(0,1) \subset \mathcal{H} \times \mathcal{H}$ is the open unit ball in $\mathcal{H} \times \mathcal{H}$. Hence,

$$
\left\|x_{1}-x_{2}\right\|+\left\|x_{1}^{\prime}-x_{2}^{\prime}\right\| \leq \ell\left\|p_{1}-p_{2}\right\|
$$

where $x_{1} \in C\left(p_{1}\right)$ and $x_{2} \in C\left(p_{2}\right), x_{1}^{\prime} \in N\left(x_{1} ; C\left(p_{1}\right)\right), x_{2}^{\prime} \in N\left(x_{2} ; C\left(p_{2}\right)\right)$, which implies (a) and (b). The converse implication is immediate.

Remark 5 Let us note that in (24) we use the norm $\|\cdot\|_{1}$ in the Cartesian product $\mathcal{H} \times \mathcal{H}$. Clearly, any other equivalent norm can be used at this point.

Let $\bar{p} \in \mathcal{D}, \bar{v} \notin C(\bar{p})$ and $\bar{x}=P(\bar{v}, \bar{p})$. From [7, Theorem 6.41] (see also [29]) the following representation holds

$$
\bar{v}-\bar{x}=\sum_{i \in I_{\bar{p}}(\bar{x})} \hat{\lambda}_{i} g_{i}(\bar{p}) \quad \text { with } \hat{\lambda}_{i} \geq 0 \text { for } i \in I_{\bar{p}}(\bar{x}) \cap I_{2} .
$$

In the proposition below we give sufficient conditions for the graphical subdifferential mapping Gr given by (6) to be Lipschitz-like at $(\bar{p}, \bar{x}, \bar{v}-\bar{x})$.

Proposition 9 Let $\bar{p} \in \mathcal{D}, \bar{v} \notin C(\bar{p})$. Assume that there exists an equivalent stable representation $\mathcal{R}$ of $\mathbb{C}$ at $(\bar{p}, \bar{v}, P(\bar{v}, \bar{p}))$, given by (3), (with set $\bar{K}$ ) and RCRCQ holds for $\mathcal{R}$ at $(\bar{p}, P(\bar{v}, \bar{p}))$ Then the graphical subdifferential mapping $G r$, given by (6), is Lipschitz-like at $(\bar{p}, P(\bar{v}, \bar{p}), \bar{v}-P(\bar{v}, \bar{p}))$.

Proof Let $\varepsilon>0$. Let $U(\bar{p}), V(0)$ be as in Corollary 1 . We have

$$
\bar{v}-P(\bar{v}, \bar{p})=\sum_{i \in \tilde{I}_{1} \cup \bar{K}} \bar{\lambda}_{i} \tilde{g}_{i}(\bar{p}), \bar{\lambda}_{i}>0, i \in \bar{K} \subset I_{\bar{p}}^{\mathcal{R}}(\bar{x}) \cap \tilde{I}_{2},
$$

where $\tilde{g}_{i}(\bar{p}), i \in \tilde{I}_{1} \cup \bar{K}$ are linearly independent.

Now, let $p_{1} \in U(\bar{p})$ and $x_{1} \in(P(\bar{v}, \bar{p})+V(0)) \cap C\left(p_{1}\right), x_{1}^{\prime} \in N\left(x_{1} ; C\left(p_{1}\right)\right) \cap$ $(\bar{v}-P(\bar{v}, \bar{p})+V(0))$. By Corollary 1 , there exists $\tilde{L} \subset I_{\bar{p}}^{\mathcal{R}}(P(\bar{v}, \bar{p})) \cap \tilde{I}_{2} \backslash \bar{K}$ such that $x_{1} \in R_{\bar{K}, \tilde{L}}\left(p_{1}\right)$ and 


$$
\begin{aligned}
& x_{1}^{\prime}=\sum_{i \in \tilde{I}_{1} \cup \bar{K}} \lambda_{i}^{1} g_{i}\left(p_{1}\right)+\sum_{i \in \tilde{L}} \lambda_{i}^{1} \tilde{g}_{i}\left(p_{1}\right), \\
& \text { where } \bar{\lambda}_{i}-\varepsilon \leq \lambda_{i}^{1} \leq \bar{\lambda}_{i}+\varepsilon, \quad i \in \tilde{I}_{1} \cup \bar{K}, \\
& 0<\lambda_{i}^{1} \leq \varepsilon, \quad i \in \tilde{L},
\end{aligned}
$$

and for any $p_{2} \in U(\bar{p})$ there exists $x_{2} \in R_{\bar{K}, \tilde{L}}\left(p_{2}\right) \subset C\left(p_{2}\right)$ such that

$$
\left\|x_{1}-x_{2}\right\| \leq \ell^{1}\left\|p_{1}-p_{2}\right\|
$$

for some $\ell^{1}>0$. Since $x_{2} \in R_{\bar{K}, \tilde{L}}\left(p_{2}\right)$ we have

$$
x_{2}^{\prime}:=\sum_{i \in \tilde{I}_{1} \cup \bar{K} \cup \tilde{L}} \lambda_{i}^{1} \tilde{g}_{i}\left(p_{2}\right) \in N\left(x_{2} ; R_{\bar{K}, \tilde{L}}\left(p_{2}\right)\right) .
$$

Then

$$
\begin{aligned}
& \left\|x_{1}^{\prime}-x_{2}^{\prime}\right\|=\left\|\sum_{i \in \tilde{I}_{1} \cup \bar{K} \cup \tilde{L}} \lambda_{i}^{1} \tilde{g}_{i}\left(p_{1}\right)-\sum_{i \in \tilde{I}_{1} \cup \bar{K} \cup \tilde{L}} \lambda_{i}^{1} \tilde{g}_{i}\left(p_{2}\right)\right\| \\
& \leq \sum_{i \in \tilde{I}_{1} \cup \bar{K} \cup \tilde{L}}\left|\lambda_{i}^{1}\right|\left\|\tilde{g}_{i}\left(p_{1}\right)-\tilde{g}_{i}\left(p_{2}\right)\right\| \leq \sum_{i \in \tilde{I}_{1} \cup \bar{K} \cup \tilde{L}}\left|\lambda_{i}^{1}\right| \ell_{\tilde{g}_{i}}\left\|p_{1}-p_{2}\right\| \\
& \leq \sum_{i \in \tilde{I}_{1} \cup \bar{K} \cup \tilde{L}}\left(\left|\bar{\lambda}_{i}\right|+\varepsilon\right) \ell_{\tilde{g}_{i}}\left\|p_{1}-p_{2}\right\| \leq \ell^{2}\left\|p_{1}-p_{2}\right\|,
\end{aligned}
$$

where we put $\ell^{2}:=\sum_{i \in I_{\bar{p}}^{\mathcal{R}}(P(\bar{v}, \bar{p}))}\left(\left|\bar{\lambda}_{i}\right|+\varepsilon\right) \ell_{\tilde{g}_{i}}$.

Now we are ready to establish our main theorem.

Theorem 6 Let $\mathcal{H}$ be a Hilbert space and let $\mathcal{D} \subset \mathcal{G}$ be a nonempty set of a normed space $\mathcal{G}$ and $\bar{p} \in \mathcal{D}$. Let $\mathbb{C}: \mathcal{D} \rightrightarrows \mathcal{H}$ be as in (3), where $f_{i}: \mathcal{D} \rightarrow \mathbb{R}, g_{i}: \mathcal{D} \rightarrow \mathcal{H}$, $i \in I_{1} \cup I_{2} \neq \emptyset, I_{1}=\emptyset \vee\{1, \ldots, q\}, I_{2}=\emptyset \vee\{q+1, \ldots, m\}$ are locally Lipschitz on $\mathcal{D}$. Let $\bar{v} \notin C(\bar{p})$. Assume that there exists an equivalent representation $\mathcal{R}$ of $\mathbb{C}$ such that

(1) RCRCQ holds for multifunction $\mathcal{R}$ at $(\bar{p}, \bar{x})$,

(2) $\mathcal{R}$ is a stable representation of $\mathbb{C}$ at $(\bar{p}, \bar{v}, \bar{x})$ with some set $\bar{K} \subset I_{\bar{p}}^{\mathcal{R}}(\bar{x}) \cap \tilde{I}_{2}$.

There exist neighbourhoods $W(\bar{v}), U(\bar{p})$ such that the Lipschitzian estimate

$$
\left\|\left(v_{1}-v_{2}\right)-2\left[P\left(v_{1}, p_{1}\right)-P\left(v_{2}, p_{2}\right)\right]\right\| \leq\left\|v_{1}-v_{2}\right\|+\ell^{0}\left\|p_{1}-p_{2}\right\|
$$

holds for all $\left(v_{1}, p_{1}\right),\left(v_{2}, p_{2}\right) \in W(\bar{v}) \times U(\bar{p})$ with some positive constant $\ell^{0}$.

In particular, we get the following result. 
Theorem 7 Let $\mathcal{H}$ be a Hilbert space and let $\mathcal{D} \subset \mathcal{G}$ be a nonempty set of a normed space $\mathcal{G}$ and $\bar{p} \in \mathcal{D}$. Let $\mathbb{C}: \mathcal{D} \rightrightarrows \mathcal{H}$ be as in (3), where $f_{i}: \mathcal{D} \rightarrow \mathbb{R}, g_{i}: \mathcal{D} \rightarrow \mathcal{H}$, $i \in I_{1} \cup I_{2} \neq \emptyset, I_{1}=\emptyset \vee\{1, \ldots, q\}, I_{2}=\emptyset \vee\{q+1, \ldots, m\}$ are locally Lipschitz on $\mathcal{D}$. Let $\bar{v} \notin C(\bar{p})$. Assume that

(1) RCRCQ holds for multifunction $\mathbb{C}$ at $(\bar{p}, \bar{x})$,

(2) $\mathbb{C}$ is a stable representation at $(\bar{p}, \bar{v}, \bar{x})$ with some set $\bar{K} \subset I_{\bar{p}}(\bar{x}) \cap \tilde{I}_{2}$.

There exist neighbourhoods $W(\bar{v}), U(\bar{p})$ such that the Lipschitzian estimate

$$
\left\|\left(v_{1}-v_{2}\right)-2\left[P\left(v_{1}, p_{1}\right)-P\left(v_{2}, p_{2}\right)\right]\right\| \leq\left\|v_{1}-v_{2}\right\|+\ell^{0}\left\|p_{1}-p_{2}\right\|
$$

holds for all $\left(v_{1}, p_{1}\right),\left(v_{2}, p_{2}\right) \in W(\bar{v}) \times U(\bar{p})$ with some positive constant $\ell^{0}$.

Proof of Theorem 6 The proof follows directly from Theorem 1, Theorem 4 and Proposition 9.

Clearly, by (25),

$$
\left\|P\left(v_{1}, p_{1}\right)-P\left(v_{2}, p_{2}\right)\right\| \leq\left\|v_{1}-v_{2}\right\|+\frac{\ell^{0}}{2}\left\|p_{1}-p_{2}\right\| .
$$

If the multifunction $\mathbb{C}$ is constant around $\bar{p}$, then assumptions of Theorem 6 are satisfied.

Corollary 2 Under assumptions of Theorem 6, projection of a given fixed $\bar{v}$ onto $C(p)$, $p \in \mathcal{D}$, i.e.,

$$
P_{\bar{v}}(p):=P(\bar{v}, p), \quad p \in \mathcal{D}
$$

is locally Lipschitz at $\bar{p}$.

Example 4 shows that for a given representation of $\mathbb{C}$ one can not expect that there exists $\bar{K}$ such that (20) and (21) holds.

Example 4 Let $\mathcal{H}=\mathbb{R}^{2}, \mathcal{G}=\mathbb{R}, \bar{p}=0, \bar{v}=(1,1)$ and

$$
\mathbb{C}(p)=\left\{\begin{array}{l|l}
x \in \mathbb{R}^{2} & \begin{array}{l}
\langle x \mid(1,0)\rangle \leq 0 \\
\langle x \mid(0,1)\rangle \leq 0 \\
\langle x \mid(1,1)\rangle \leq p
\end{array}
\end{array}\right\}
$$

In this case we have

$$
P(\bar{v}, p)= \begin{cases}(0,0) & \text { if } p \geq 0 \\ \left(\frac{p}{2}, \frac{p}{2}\right) & \text { if } p<0\end{cases}
$$

and $P(\bar{v}, p)$ is a Lipschitz function of $p$. On the other hand,

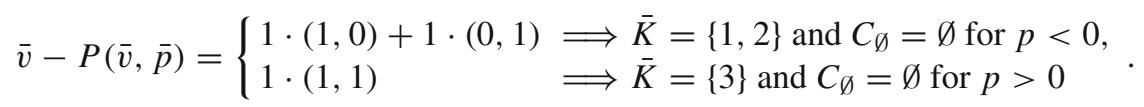

Hence, the representation (27) of $\mathbb{C}$ is not stable at $(0,(0,0),(1,1))$ (see Fig. 1). 


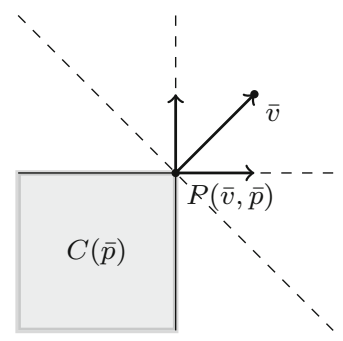

(a) $\bar{p}=0$

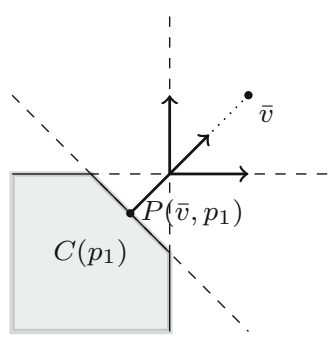

(b) $p_{1}<0$

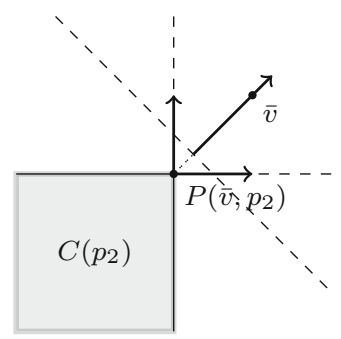

(c) $p_{2}>0$

Fig. 1 Illustration of the set $C(\cdot)$ given by $(27)$ and projection $P(\bar{v}, \cdot)$ under different choice of parameters $p$

Remark 6 Let us note that multifunction $\mathbb{C}$, given by (27), can be equivalently represented as

$$
\mathbb{C}(p)=\left\{\begin{array}{l|l}
x \in \mathbb{R}^{2} & \begin{array}{l}
\langle x \mid(1,0)\rangle \leq 0 \\
\langle x \mid(0,1)\rangle \leq 0 \\
\langle x|(1,1) \leq g_{3}(p)
\end{array}
\end{array}\right\},
$$

where

$$
g_{3}(p)=\left\{\begin{array}{l}
p \text { if } p<0 \\
0 \text { if } p \geq 0
\end{array} .\right.
$$

The representation given in (28) is stable at $(\bar{p}, \bar{v}, P(\bar{v}, \bar{p}))$ and RCRCQ holds at $(\bar{p}, P(\bar{p}, \bar{v}))$.

Corollary 3 Suppose that in the definition of the set $C(p), p \in \mathcal{D}$, given in (3), $I_{2}=\emptyset$, i.e.,

$$
C(p):=\left\{x \in \mathcal{H} \mid\left\langle x \mid g_{i}(p)\right\rangle=f_{i}(p), i \in I_{1}\right\} .
$$

Let $\bar{p} \in \mathcal{D}, \bar{v} \notin C(\bar{p}), \bar{x}=P(\bar{v}, \bar{p})$ and the following hold:

(1) RCRCQ holds for multifunction $\mathbb{C}$ at $(\bar{p}, \bar{x})$, i.e., there exists a neighbourhood $U(\bar{p})$ such that

$$
\operatorname{rank}\left\{g_{i}(p), i \in I_{1}\right\}=\operatorname{rank}\left\{g_{i}(\bar{p}), i \in I_{1}\right\}, \quad p \in U(\bar{p}) .
$$

(2) $\bar{x} \in \liminf _{p \rightarrow \bar{p}, p \in \mathcal{D}} \mathbb{C}(p)$.

Then the projection $P(v, p)$ is locally Lipschitz at $(\bar{v}, \bar{p})$.

When LICQ condition holds for set $C(\bar{p})$ at $P(\bar{v}, \bar{p})$, i.e., when $g_{i}(\bar{p}), i \in I_{\bar{p}}(P(\bar{v}, \bar{p}))$ are linearly independent, Theorem 7 can rewritten in a considerably simplified form. 
Theorem 8 Let $\mathcal{H}$ be a Hilbert space and let $\mathcal{D} \subset \mathcal{G}$ be a nonempty set of a normed space $\mathcal{G}$ and $\bar{p} \in \mathcal{D}$. Let $\mathbb{C}: \mathcal{D} \rightrightarrows \mathcal{H}$ be as in (3), where $f_{i}: \mathcal{D} \rightarrow \mathbb{R}, g_{i}: \mathcal{D} \rightarrow \mathcal{H}$, $i \in I_{1} \cup I_{2} \neq \emptyset, I_{1}=\emptyset \vee\{1, \ldots, q\}, I_{2}=\{q+1, \ldots, m\} \vee \emptyset$ are locally Lipschitz on D. Let $\bar{v} \notin C(\bar{p})$ and LICQ hold for set $C(\bar{p})$ at $P(\bar{v}, \bar{p})$. There exist neighbourhoods $W(\bar{v}), U(\bar{p})$ such that the Lipschitzian estimate

$$
\left\|\left(v_{1}-v_{2}\right)-2\left[P\left(v_{1}, p_{1}\right)-P\left(v_{2}, p_{2}\right)\right]\right\| \leq\left\|v_{1}-v_{2}\right\|+\ell^{0}\left\|p_{1}-p_{2}\right\|
$$

holds for all $\left(v_{1}, p_{1}\right),\left(v_{2}, p_{2}\right) \in W(\bar{v}) \times U(\bar{p})$ with some positive constant $\ell^{0}$.

Proof We have

$$
\bar{v}-P(\bar{v}, \bar{p})=\sum_{i \in I_{1} \cup \bar{K}} \bar{\lambda}_{i} g_{i}(\bar{p}), \bar{\lambda}_{i}>0, i \in \bar{K} \subset I_{\bar{p}}(\bar{x}) \cap I_{2},
$$

where $g_{i}(\bar{p}), \bar{p} \in \mathcal{D}, i \in I_{1} \cup K \subset I_{\bar{p}}(P(\bar{v}, \bar{p}))$ are linearly independent. Thus (1) of Theorem 7 is satisfied.

Now we show (2) of Theorem 7. Observe that LICQ hold for set $C_{\bar{K}, L}(\bar{p})$ at $P(\bar{v}, \bar{p})$ with any $L$ satisfying (21). Hence, MFCQ holds for set $C_{\bar{K}, L}(\bar{p})$ at $P(\bar{v}, \bar{p})$ with any $L$ satisfying (21). Thus, by Theorem 2.87 of [6], for any $L$ satisfying (21), there exist $\alpha>0$ and a neighbourhood $U(\bar{p})$ of $\bar{p}$ such that for all $p \in U(\bar{p})$

$$
\begin{aligned}
& \operatorname{dist}\left(\bar{x}, C_{\bar{K}, L}(p)\right) \leq \alpha\left(\sum_{i \in I_{1} \cup \bar{K} \cup L}\left|\left\langle\bar{x} \mid g_{i}(p)\right\rangle-f_{i}(p)\right|\right.
\end{aligned}
$$

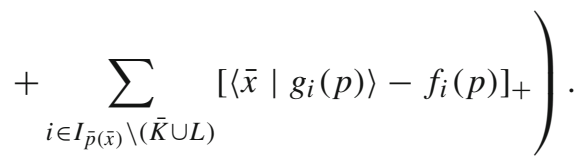

This implies that $\bar{x} \in \liminf _{p \rightarrow \bar{p}, p \in \mathcal{D}} \mathbb{C}_{\bar{K}, L}(p)$ for any $L$ satisfying (21), i.e. assumption (2) of Theorem 7 is satisfied, which proves the assertion.

In view of proof of Theorem 8 the following corollary holds.

Corollary 4 Let $\mathcal{H}$ be a Hilbert space and let $\mathcal{D} \subset \mathcal{G}$ be a nonempty set of a normed space $\mathcal{G}$ and $\bar{p} \in \mathcal{D}$. Let $\mathbb{C}: \mathcal{D} \rightrightarrows \mathcal{H}$ be as in (3), where $f_{i}: \mathcal{D} \rightarrow \mathbb{R}, g_{i}: \mathcal{D} \rightarrow \mathcal{H}$, $i \in I_{1} \cup I_{2} \neq \emptyset, I_{1}=\emptyset \vee\{1, \ldots, q\}, I_{2}=\emptyset \vee\{q+1, \ldots, m\}$ are locally Lipschitz on D. Let $\bar{v} \notin C(\bar{p})$ and

$$
\bar{v}-P(\bar{v}, \bar{p})=\sum_{i \in I_{1} \cup \bar{K}} \bar{\lambda}_{i} g_{i}(\bar{p}), \bar{\lambda}_{i}>0, i \in \bar{K} \subset I_{\bar{p}}(\bar{x}) \cap I_{2},
$$

where $g_{i}(\bar{p}), i \in I_{1} \cup \bar{K}$ are linearly independent. Assume that

(1) RCRCQ holds for multifunction $\mathbb{C}$ at $(\bar{p}, \bar{x})$,

(2) MFCQ holds for set $C_{\bar{K}, L}(\bar{p})$ at $P(\bar{v}, \bar{p})$ with any $L$ satisfying (21). 
There exist neighbourhoods $W(\bar{v}), U(\bar{p})$ such that the Lipschitzian estimate

$$
\left\|\left(v_{1}-v_{2}\right)-2\left[P\left(v_{1}, p_{1}\right)-P\left(v_{2}, p_{2}\right)\right]\right\| \leq\left\|v_{1}-v_{2}\right\|+\ell^{0}\left\|p_{1}-p_{2}\right\|
$$

holds for all $\left(v_{1}, p_{1}\right),\left(v_{2}, p_{2}\right) \in W(\bar{v}) \times U(\bar{p})$ with some positive constant $\ell^{0}$.

\section{Conclusion}

In the present paper we proved Lipschitzian stability of projections (in the sense of (25)) onto parametric polyhedral sets in Hilbert space setting with parameters appearing both in left- and right-hand sides of constraints, which are assumed to be locally Lipschitz. The equality and inequality constraints are allowed. Basic tools for our main results are RCRCQ condition and the representation stability condition (see Definition 5).

In general, there is no relationship between RCRCQ and MFCQ (cf. [15]). Moreover, in Propositions 5, 7, Corollary 1, Theorem 6 the conclusions depend upon formula (12) and the representation stability condition in which the index set $\bar{K}$ may not be uniquely defined.

Open Access This article is licensed under a Creative Commons Attribution 4.0 International License, which permits use, sharing, adaptation, distribution and reproduction in any medium or format, as long as you give appropriate credit to the original author(s) and the source, provide a link to the Creative Commons licence, and indicate if changes were made. The images or other third party material in this article are included in the article's Creative Commons licence, unless indicated otherwise in a credit line to the material. If material is not included in the article's Creative Commons licence and your intended use is not permitted by statutory regulation or exceeds the permitted use, you will need to obtain permission directly from the copyright holder. To view a copy of this licence, visit http://creativecommons.org/licenses/by/4.0/.

\section{Appendix}

Lemma 1 [4, Lemma 10] Let $J=\{1, \ldots, k\}$. Let $g_{i}: \mathcal{H} \rightarrow \mathcal{H}, i \in J$ be continuous operators and let $\bar{p}$ be such that $g_{i}(\bar{p}), i \in J$ are linearly independent. Then there exists a neighbourhood $U(\bar{p})$ such that for all $p \in U(\bar{p}), g_{i}(p), i \in J$ are linearly independent.

Lemma 2 [7, Lemma 6.33] If a nonzero vector $x$ is a positive linear combination of the nonzero vectors $g_{1}, \ldots, g_{n}$, then $x$ is a positive linear combination of linearly independent subset of $\left\{g_{1}, \ldots, g_{n}\right\}$.

Remark 7 Let $J=\{1, \ldots, k\}, J=W_{1} \cup W_{2}, W_{1} \cap W_{2}=\emptyset$ and let $x=\sum_{i \in J} \lambda_{i} g_{i}$, $\lambda_{i} \leq 0, i \in W_{1}, \lambda_{i} \geq 0, i \in W_{2}$, where $g_{i}, i \in J$ are nonzero vectors. Then there exists $\bar{J} \subset J$ and $\bar{\lambda}_{i}, \bar{i} \in \bar{J}$ such that

$$
x=\sum_{i \in \bar{J}} \lambda_{i} g_{i}, \quad \lambda_{i}<0, i \in \bar{J} \cap W_{1}, \quad \lambda_{i}>0, i \in \bar{J} \cap W_{2}
$$

and $g_{i}, i \in \bar{J}$ are linearly independent. 
Proof We have $x=\sum_{i \in J_{1}} \lambda_{i} g_{i}$, where $J_{1} \subset J$ and $\lambda_{i}<0, i \in J_{1} \cap W_{1}, \lambda_{i}>0$, $i \in J_{1} \cap W_{2}$. Let

$$
\tilde{g}_{i}=\left\{\begin{array}{r}
g_{i} \text { if } i \in W_{2} \\
-g_{i} \text { if } i \in W_{1}
\end{array} \quad i \in J_{1}, \quad \tilde{\lambda}_{i}=\left\{\begin{array}{r}
\lambda_{i} \text { if } i \in W_{2} \\
-\lambda_{i} \text { if } i \in W_{1}
\end{array} \quad i \in J_{1} .\right.\right.
$$

Then $x=\sum_{i \in J_{1}} \tilde{\lambda}_{i} \tilde{g}_{i}$ and $\tilde{\lambda}_{i}, i \in J_{1}$ are positive. Applying Lemma 2 we have that there exists $\bar{J} \subset J_{1}$ and $\hat{\lambda}_{i}>0, i \in \bar{J}$ such that $x=\sum_{i \in \bar{J}} \hat{\lambda}_{i} \tilde{g}_{i}$ and $\tilde{g}_{i}, i \in \bar{J}$ are linearly independent. Now let

$$
\bar{\lambda}_{i}=\left\{\begin{aligned}
\hat{\lambda}_{i} & \text { if } i \in W_{2} \\
-\hat{\lambda}_{i} & \text { if } i \in W_{1}
\end{aligned} \quad i \in \bar{J}\right.
$$

Then $x=\sum_{i \in \bar{J}} \bar{\lambda}_{i} g_{i}, \bar{\lambda}_{i}<0, i \in \bar{J} \cap W_{1}, \bar{\lambda}_{i}>0, i \in \bar{J} \cap W_{2}$.

Lemma 3 [4, Lemma 12] Let $x=\sum_{i \in J_{1}} \lambda_{i} a_{i}+\sum_{i \in J_{2}} \lambda_{i} a_{i}, J_{1} \cap J_{2}=\emptyset, J_{1}, J_{2}$ finite sets, $\lambda_{i} \in \mathbb{R}, i \in J_{1}, \lambda_{i} \geq 0, i \in J_{2}$ and $a_{i}, i \in J_{1} \cup J_{2}$ are non-zero vectors. Assume that $a_{i}, i \in J_{1}$ are linearly independent. Then there exists $J_{2}^{\prime} \subset J_{2}$ and $\lambda_{i}^{\prime}, i \in J_{1} \cup J_{2}^{\prime}$, $\lambda_{i}^{\prime} \in \mathbb{R}, i \in J_{1}, \lambda_{i}^{\prime}>0, i \in J_{2}^{\prime}$ such that

$$
\sum_{i \in J_{1}} \lambda_{i} a_{i}+\sum_{i \in J_{2}} \lambda_{i} a_{i}=\sum_{i \in J_{1}} \lambda_{i}^{\prime} a_{i}+\sum_{i \in J_{2}^{\prime}} \lambda_{i}^{\prime} a_{i}
$$

and $a_{i}, i \in J_{1} \cup J_{2}^{\prime}$ are linearly independent.

Lemma 4 Let $\mathcal{H}$ be a Hilbert space. Let $\left\{u_{i}^{n}\right\}_{n \in \mathbb{N}}, i \in 1, \ldots, K$ be a sequence of $K$-tuples of vectors from $\mathcal{H}$ such that for any $n \in \mathbb{N}, u_{i}^{n}, i \in 1, \ldots, K$ are linearly independent. Assume that

(1) $u_{i}^{n} \rightarrow u_{i}$, for $i=1 \ldots, K$, where $u_{i}, i=1 \ldots, K$, are linearly independent,

(2) $\sum_{i=1}^{K} \lambda_{i}^{n} u_{i}^{n} \rightarrow \sum_{i=1}^{K} \bar{\lambda}_{i} u_{i}$, where $\lambda_{i}^{n}, \bar{\lambda}_{i} \in \mathbb{R}, i=1, \ldots K, n \in \mathbb{N}$.

Then $\lambda_{i}^{n} \rightarrow \bar{\lambda}_{i}, i=1, \ldots, K$.

Proof For any $n \in \mathbb{N}$ let $\left\{e_{i}^{n}\right\}_{n \in \mathbb{N}} i=1, \ldots, K$ be a sequence of orthogonal vectors obtained by the Gram-Schmidt orthogonalization of $u_{i}^{n} i=1, \ldots, K$, i.e.,

$$
\begin{aligned}
& e_{1}^{n}=u_{1}^{n}, \\
& e_{k}^{n}=u_{k}^{n}-\sum_{i=1}^{k-1} \frac{\left\langle u_{k}^{n} \mid e_{i}^{n}\right\rangle}{\left\|e_{i}^{n}\right\|^{2}} e_{i}^{n}, \quad k=2, \ldots, K
\end{aligned}
$$

and $e_{i}, i=1, \ldots, K$, be orthogonal vectors obtained by the Gram-Schmidt orthogonalization of $u_{i} i=1, \ldots, K$. Since $u_{i}^{n} \rightarrow u_{i}, i=1, \ldots, K$ we have $e_{i}^{n} \rightarrow e_{i}$, $i=1, \ldots, K$. 
Let $\tilde{\lambda}_{i}^{n}, \tilde{\lambda}_{i} \in \mathbb{R}, i=1, \ldots K, n \in \mathbb{N}$ be such that $\sum_{i=1}^{K} \lambda_{i}^{n} u_{i}^{n}=\sum_{i=1}^{K} \tilde{\lambda}_{i}^{n} e_{i}^{n}$ and $\sum_{i=1}^{K} \bar{\lambda}_{i} u_{i}=\sum_{i=1}^{K} \tilde{\lambda}_{i} e_{i}$. Since $\sum_{i=1}^{K}\left(\lambda_{i}^{n} u_{i}^{n}-\bar{\lambda}_{i} u_{i}\right) \rightarrow 0$ we have

$$
\sum_{i=1}^{K}\left(\tilde{\lambda}_{i}^{n} e_{i}^{n}-\tilde{\lambda}_{i} e_{i}\right) \rightarrow 0
$$

We have

$$
\sum_{i=1}^{K}\left(\tilde{\lambda}_{i}^{n} e_{i}^{n}-\tilde{\lambda}_{i} e_{i}\right)=\sum_{i=1}^{K} \tilde{\lambda}_{i}^{n}\left(e_{i}^{n}-e_{i}\right)+\sum_{i=1}^{K}\left(\tilde{\lambda}_{i}^{n}-\tilde{\lambda}_{i}\right) e_{i}
$$

By taking scalar products with $e_{k}, k \in\{1, \ldots, K\}$ we obtain

$$
\sum_{i=1}^{K} \tilde{\lambda}_{i}^{n}\left\langle e_{i}^{n}-e_{i} \mid e_{k}\right\rangle+\left(\tilde{\lambda}_{k}^{n}-\tilde{\lambda}_{k}\right)\left\|e_{k}\right\|^{2} \rightarrow 0, \quad k=1, \ldots, K
$$

Now we show that $\tilde{\lambda}_{i}^{n}$ are bounded, i.e. $\left|\tilde{\lambda}_{i}^{n}\right| \leq M$ for $i=1, \ldots, K, n \in \mathbb{N}$ for some $M \geq 0$. We have

$$
\left\|\sum_{i=1}^{K} \tilde{\lambda}_{i}^{n} e_{i}^{n}\right\|^{2}=\sum_{i=1}^{K}\left(\tilde{\lambda}_{i}^{n}\right)^{2}\left\|e_{i}^{n}\right\|^{2} \geq\left(\tilde{\lambda}_{k}^{n}\right)^{2}\left\|e_{k}^{n}\right\|^{2} \text { for all } k \in\{1, \ldots, K\}
$$

If there exists $k \in\{1, \ldots, K\}$ such that $\left|\tilde{\lambda}_{n}^{k}\right| \rightarrow+\infty$, then $\left\|\sum_{i=1}^{K} \tilde{\lambda}_{i}^{n} e_{i}^{n}\right\|^{2} \rightarrow+\infty$, which contradicts $\left\|\sum_{i=1}^{K} \tilde{\lambda}_{i}^{n} e_{i}^{n}\right\|^{2} \rightarrow\left\|\sum_{i=1}^{K} \tilde{\lambda}_{i} e_{i}\right\|^{2}=\left\|\sum_{i=1}^{K} \lambda_{i} u_{i}\right\|^{2}<+\infty$.

Since $\tilde{\lambda}_{i}^{n}$ are bounded and $e_{i}^{n}-e_{i} \rightarrow 0, i=1, \ldots, K$ from (29) and (30) we conclude that $\tilde{\lambda}_{k}^{n} \rightarrow \tilde{\lambda}_{k}$ for any $k \in\{1,2 \ldots, K\}$.

For each $n \in \mathbb{N}$ we have

$$
\sum_{i=1}^{K} \lambda_{i}^{n} u_{i}^{n}=\sum_{i=1}^{K} \tilde{\lambda}_{i}^{n} e_{i}^{n}=\tilde{\lambda}_{1}^{n} u_{1}^{n}+\sum_{i=2}^{K} \tilde{\lambda}_{i}^{n}\left(u_{i}^{n}-\sum_{j=1}^{i-1} \frac{\left\langle u_{i}^{n} \mid e_{j}^{n}\right\rangle}{\left\|e_{j}^{n}\right\|^{2}} e_{j}^{n}\right)
$$

Since $u_{i}^{n}, i=1, \ldots, K$ are linearly independent we have

$$
\begin{aligned}
& \lambda_{K}^{n} u_{K}^{n}=\tilde{\lambda}_{K}^{n} u_{K}^{n}, \\
& \lambda_{K-1}^{n} u_{K-1}^{n}=\tilde{\lambda}_{K-1}^{n} u_{K-1}^{n}-\tilde{\lambda}_{K}^{n} \frac{\left\langle u_{K}^{n} \mid e_{K-1}^{n}\right\rangle}{\left\|e_{K-1}^{n}\right\|^{2}} u_{K-1}^{n}, \\
& \lambda_{K-2}^{n} u_{K-2}^{n}=\tilde{\lambda}_{K-2}^{n} u_{K-2}^{n}-\tilde{\lambda}_{K-1}^{n} \frac{\left\langle u_{K-1}^{n} \mid e_{K-2}^{n}\right\rangle}{\left\|e_{K-2}^{n}\right\|^{2}} u_{K-2}^{n}
\end{aligned}
$$




$$
\begin{aligned}
& +\tilde{\lambda}_{K}^{n}\left(\frac{\left\langle u_{K}^{n} \mid e_{K-1}^{n}\right\rangle}{\left\|e_{K-1}^{n}\right\|^{2}} \frac{\left\langle u_{K-1}^{n} \mid e_{K-2}^{n}\right\rangle}{\left\|e_{K-2}^{n}\right\|^{2}} u_{K-2}^{n}\right. \\
& \left.-\frac{\left\langle u_{K}^{n} \mid e_{K-2}^{n}\right\rangle}{\left\|e_{K-2}^{n}\right\|^{2}} u_{K-2}^{n}\right)
\end{aligned}
$$

Hence,

$$
\begin{aligned}
& \lambda_{K}^{n}= \tilde{\lambda}_{K}^{n}, \\
& \lambda_{K-1}^{n}=\tilde{\lambda}_{K-1}^{n}-\tilde{\lambda}_{K}^{n} \frac{\left\langle u_{K}^{n} \mid e_{K-1}^{n}\right\rangle}{\left\|e_{K-1}^{n}\right\|^{2}}, \\
& \lambda_{K-2}^{n}=\tilde{\lambda}_{K-2}^{n}-\tilde{\lambda}_{K-1}^{n} \frac{\left\langle u_{K-1}^{n} \mid e_{K-2}^{n}\right\rangle}{\left\|e_{K-2}^{n}\right\|^{2}} \\
& \quad+\tilde{\lambda}_{K}^{n}\left(\frac{\left\langle u_{K}^{n} \mid e_{K-1}^{n}\right\rangle}{\left\|e_{K-1}^{n}\right\|^{2}} \frac{\left\langle u_{K-1}^{n} \mid e_{K-2}^{n}\right\rangle}{\left\|e_{K-2}^{n}\right\|^{2}}-\frac{\left\langle u_{K}^{n} \mid e_{K-2}^{n}\right\rangle}{\left\|e_{K-2}^{n}\right\|^{2}}\right)
\end{aligned}
$$

Since $e_{i}^{n} \rightarrow e_{i}, u_{i}^{n} \rightarrow u_{i}$ and $\tilde{\lambda}_{i}^{n} \rightarrow \tilde{\lambda}_{i}$ for any $i \in\{1, \ldots, K\}$ we obtain that $\lambda_{i}^{n}$ converges for any $i \in\{1, \ldots, K\}$ and $\lambda_{i}^{n} \rightarrow \lambda_{i}, i=1, \ldots, K$.

\section{References}

1. Andreani, R., Silva, P.J.: Constant rank constraint qualifications: a geometric introduction. Pesqui. Operacional 34, 481-494 (2014)

2. Attouch, H., Wets, R.J.B.: Quantitative stability of variational systems. II. A framework for nonlinear conditioning. SIAM J. Optim. 3(2), 359-381 (1993). https://doi.org/10.1137/0803016

3. Bednarczuk, E.M., Rutkowski, K.E.: On Lipschitz-like property for polyhedral moving sets. arXiv e-prints arXiv:1811.05166 (2018). To appear in SIOPT

4. Bednarczuk, E., Rutkowski, K.: On lipschitz-like property for polyhedral moving sets. SIAM J. Optim. 29(4), 2504-2516 (2019). https://doi.org/10.1137/18M1226518

5. Bednarczuk, E.M., Minchenko, L.I., Rutkowski, K.E.: On Lipschitz-like continuity of a class of setvalued mappings. arXiv e-prints arXiv:1905.08173 (2019)

6. Bonnans, J.F., Shapiro, A.: Perturbation analysis of optimization problems. Springer Series in Operations Research. Springer, New York (2000).https://doi.org/10.1007/978-1-4612-1394-9

7. Deutsch, F.: Best approximation in inner product spaces. CMS Books in Mathematics/Ouvrages de Mathématiques de la SMC, vol. 7. Springer, New York (2001). https://doi.org/10.1007/978-1-46849298-9

8. Dontchev, A.L.: Perturbations, approximations and sensitivity analysis of optimal control systems. Lecture Notes in Control and Information Sciences, vol. 52. Springer, Berlin (1983). https://doi.org/ 10.1007/BFb0043612

9. Dontchev, A., Rockafellar, R.: Implicit functions and solution mappings. A view from variational analysis. 2nd updated ed (2014)

10. Dontchev, A.L., Quincampoix, M., Zlateva, N.: Aubin criterion for metric regularity. J. Convex Anal. 13(2), 281-297 (2006) 
11. Engelking, R.: General topology. Sigma Series in Pure Mathematics, vol. 6, second edn. Heldermann, Berlin (1989). Translated from the Polish by the author

12. Gfrerer, H., Outrata, J.V.: On the Aubin property of solution maps to parameterized variational systems with implicit constraints. Optimization 12, 11-21 (2019). https://doi.org/10.1080/02331934. 2019.1657427

13. Janin, R.: Directional derivative of the marginal function in nonlinear programming. Math. Program. Stud. 21, 110-126 (1984). https://doi.org/10.1007/bfb0121214. Sensitivity, stability and parametric analysis

14. Klatte, D., Kummer, B.: Aubin property and uniqueness of solutions in cone constrained optimization. Math. Methods Oper. Res. 77(3), 291-304 (2013). https://doi.org/10.1007/s00186-013-0429-6

15. Kruger, A.Y., Minchenko, L., Outrata, J.V.: On relaxing the Mangasarian-Fromovitz constraint qualification. Positivity 18(1), 171-189 (2014). https://doi.org/10.1007/s11117-013-0238-4

16. Lu, S.: Implications of the constant rank constraint qualification. Math. Program. 126(2, Ser. A), 365-392 (2011). https://doi.org/10.1007/s10107-009-0288-3

17. Lu, S.: Relation between the constant rank and the relaxed constant rank constraint qualifications. Optimization 61(5), 555-566 (2012). https://doi.org/10.1080/02331934.2010.527972

18. Minchenko, L.I., Sakolchik, P.P.: Hölder behavior of optimal solutions and directional differentiability of marginal functions in nonlinear programming. J. Optim. Theory Appl. 90(3), 555-580 (1996). https://doi.org/10.1007/BF02189796

19. Minchenko, L., Stakhovski, S.: On relaxed constant rank regularity condition in mathematical programming. Optimization 60(4), 429-440 (2011). https://doi.org/10.1080/02331930902971377

20. Minchenko, L., Stakhovski, S.: Parametric nonlinear programming problems under the relaxed constant rank condition. SIAM J. Optim. 21(1), 314-332 (2011). https://doi.org/10.1137/090761318

21. Mordukhovich, B.S.: Variational analysis and generalized differentiation. I. Grundlehren der Mathematischen Wissenschaften [Fundamental Principles of Mathematical Sciences], vol. 330. Springer, Berlin (2006). Basic theory

22. Mordukhovich, B.S.: Variational analysis and applications. Springer Monographs in Mathematics. Springer, Cham (2018). https://doi.org/10.1007/978-3-319-92775-6

23. Mordukhovich, B.S., Nghia, T.T.A.: Full Lipschitzian and Hölderian stability in optimization with applications to mathematical programming and optimal control. SIAM J. Optim. 24(3), 1344-1381 (2014). https://doi.org/10.1137/130906878

24. Mordukhovich, B.S., Rockafellar, R.T., Sarabi, M.E.: Characterizations of full stability in constrained optimization. SIAM J. Optim. 23(3), 1810-1849 (2013). https://doi.org/10.1137/120887722

25. Mordukhovich, B.S., Nghia, T.T.A., Pham, D.T.: Full stability of general parametric variational systems. Set-Valued and Variational Analysis (2018). https://doi.org/10.1007/s11228-018-0474-7

26. Outrata, J., Ramírez, C.H.: On the Aubin property of critical points to perturbed second-order cone programs. SIAM J. Optim. 21(3), 798-823 (2011). https://doi.org/10.1137/100807168

27. Ralph, D., Dempe, S.: Directional derivatives of the solution of a parametric nonlinear program. Math. Program. 70(2, Ser. A), 159-172 (1995). https://doi.org/10.1007/BF01585934

28. Rockafellar, R.T., Wets, R.J.B.: Variational analysis. Grundlehren der Mathematischen Wissenschaften [Fundamental Principles of Mathematical Sciences], vol. 317. Springer, Berlin (1998). https://doi.org/ 10.1007/978-3-642-02431-3

29. Rutkowski, K.E.: Closed-form expressions for projectors onto polyhedral sets in hilbert spaces. SIAM J. Optim. 27(3), 1758-1771 (2017). https://doi.org/10.1137/16M1087540

30. Yen, N.D.: Lipschitz continuity of solutions of variational inequalities with a parametric polyhedral constraint. Math. Oper. Res. 20(3), 695-708 (1995). http://www.jstor.org/stable/3690178

31. Zarantonello, E.H.: Projections on convex sets in Hilbert space and spectral theory. I. Projections on convex sets. In: Contributions to nonlinear functional analysis (Proc. Sympos., Math. Res. Center, Univ. Wisconsin, Madison), pp. 237-341 (1971)

Publisher's Note Springer Nature remains neutral with regard to jurisdictional claims in published maps and institutional affiliations. 